INRA Prod. Anim., 2017, 30 (2), 107-124

\title{
La sélection génétique des races bovines allaitantes en France : un dispositif et des outils innovants au service des filières viande
}

\author{
L. GRIFFON ${ }^{1}$, P. BOULESTEIX ${ }^{2}$, A. DELPEUCH ${ }^{2}$, A. GOVIGNON-GION ${ }^{3,8}, J$. GUERRIER ${ }^{4}$,

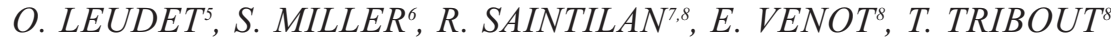 \\ I Institut de l'Elevage, 149 rue de Bercy, 75595, Paris, France \\ 2 Institut de l'Elevage, Boulevard des Arcades, 87060, Limoges, France \\ ${ }^{3}$ Institut de l'Elevage, GABI, domaine de Vilvert, 78350, Jouy-en-Josas, France \\ ${ }^{4}$ Institut de l'Elevage, 9, allée Pierre de Fermat, 63170, Aubière, France \\ ${ }_{5}^{5}$ Institut de l'Elevage, 9, rue André Brouard, CS 70510, 49105, Angers, France \\ ${ }^{6}$ Institut de l'Elevage, 25, boulevard Léon Blum, 58000, Nevers, France \\ 7 Allice, GABI, domaine de Vilvert, 78350, Jouy-en-Josas, France \\ ${ }^{8}$ GABI, AgroParisTech, INRA, Université Paris-Saclay, 78350, Jouy-en-Josas, France \\ Courriel:laurent.griffon@idele.fr
}

Depuis ses premières valorisations en 1993, l'évaluation génétique en ferme dite " IBOVAL $»^{1}$ n'a eu de cesse d'évoluer pour valoriser sous forme d'index le plus grand nombre de phénotypes observés chez les bovins allaitants. La sélection des mâles les plus prometteurs dans les outils (stations, testage) est permise par la fourniture d'index fiables. Depuis 2015, l'outil génomique est déployé en races Charolaise, Limousine et Blonde d'Aquitaine.

La sélection des bovins allaitants se caractérise par son double objectif avec le tri des meilleurs individus à la fois sur leur potentiel de croissance et de production de viande, mais aussi sur leurs qualités maternelles. Pour ces dernières, il s'agit de disposer de femelles aptes à se reproduire régulièrement et à élever dans les meilleures conditions leurs veaux jusqu'au sevrage. Pour atteindre ce double objectif, plusieurs types d'outils ont été développés en France, tant pour recueillir les informations issues des troupeaux de vaches, que pour enregistrer les performances bouchères des mâles.

Contrairement à l'Allemagne (troupeau à dominante laitière) ou à l'Irlande (forte utilisation du croisement), la production de viande bovine en France se caractérise par l'élevage de vaches allaitantes exploitées essentiellement en race pure $(83,9 \%$ des naissances en 2015, Institut de l'Élevage 2017), qui sont à l'origine de $65 \%$ des viandes issues de gros bovins (Institut de l'Élevage 2016). Cette particularité a conduit la France à développer le contrôle de performances en race pure sur de grands effectifs, ce qui a permis la mise en place d'évaluations génétiques dans 10 races : Charolaise, Limousine, Blonde d'Aquitaine, Aubrac, Salers,
Parthenaise, Rouge des Prés, Gasconne, Bazadaise et INRA 95.

L'objet de cet article est de faire un tour d'horizon de la sélection des bovins allaitants en France. Un inventaire des outils de collecte et des informations disponibles est tout d'abord fait. Puis, la valorisation de ces informations au travers des évaluations génétiques est présentée. La construction des index de synthèse multi-caractères (traduisant les objectifs de sélection) ainsi que la gestion des particularités génétiques sont ensuite décrites. Enfin, l'impact des programmes de sélection et les perspectives sont évoqués.

\section{1 / Informations disponibles pour l'évaluation génétique des reproducteurs en bovins allaitants}

Le cheptel bovin allaitant français compte 4158000 vaches et 93000 détenteurs de plus de 5 vaches (Institut de l'Élevage 2016). Ce cheptel de vaches allaitantes représente $53 \%$ des effectifs totaux de vaches. Les principales races élevées sont : les races Charolaise (1 529000 vaches, $37 \%$ des vaches allaitantes), Limousine (1 089000 vaches, 26\%), Blonde d'Aquitaine (494 000 vaches, $12 \%)$, Salers (215 000 vaches, $5 \%)$ et Aubrac (190 000 vaches, 5\%).

La population bovine allaitante est conduite en race pure, le croisement étant peu utilisé. Cependant, le pourcentage de veaux croisés varie fortement entre les races (Institut de l'Élevage 2017) : il est faible en Parthenaise (2,2\%), en Charolaise $(5,7 \%)$ et en Rouge des Prés $(10,9 \%)$, mais atteint $28,6,32,7$ et $57,4 \%$ respectivement dans les races Gasconne, Aubrac et Salers. Les croisements sont majoritairement assurés par des taureaux Charolais (48\%), Limousins $(27 \%)$ et Blonds d'Aquitaine (12\%).

Dans cet article, nous utiliserons indifféremment les termes "phénotype » et « performance » pour désigner les mesures collectées sur des caractères d'intérêt.

La collecte des informations nécessaires à l'évaluation génétique des reproducteurs en bovins allaitants repose sur différents outils :

i) la Certification de la Parenté Bovine $(\mathrm{CPB})^{1}$ et le contrôle de performances

${ }^{1}$ Voir glossaire à la fin de l'article. L'ensemble des abréviations utilisées dans cet article sont récapitulées dans le glossaire. 
en ferme qui mettent en œuvre une importante collecte dans les élevages volontaires, servant également de base au contrôle sur descendance en ferme ;

ii) les Stations d'Évaluation (SE) et de Contrôle Individuel (CI) destinées à la sélection des mâles reproducteurs ;

iii) le contrôle sur descendance en station, destiné à évaluer précisément la valeur des taureaux d'Insémination Animale (IA).

La CPB et le contrôle de performances induisent une stratification des élevages. Les élevages volontaires sont tout d'abord adhérents au service CPB proposé par les Établissements d'Élevage (un peu plus de 21000 élevages en 2015 , soit $23 \%$, figure 1). Un peu plus de 7600 d'entre eux ( $8 \%$ du total) adhèrent en plus à un organisme « Bovins Croissance » et participent au contrôle de performances en ferme selon un protocole incluant des pesées des veaux, dit « formule VA4 ». Les organismes « Bovins Croissance » proposent également un service d'appui technique sans pesée de veaux aux adhérents de la CPB ( formule VA0 »). Les élevages adhérant à ces 3 niveaux de service (CPB, services VA0 et VA4) constituent la « base de sélection » du dispositif bovin allaitant.

Ci-dessous, la collecte faite à différents stades au cours de la vie des animaux
Encadré. Quelques périodes clés de la sélection génétique bovine allaitante.

i) De 1955 à 1975 : mise en place des outils de sélection des taureaux d'insémination artificielle (stations de contrôle individuel et sur descendance) et du contrôle de croissance en ferme.

ii) De 1975 à 1990 : développement de l'évaluation génétique des vaches allaitantes, mise en place des stations d'évaluation.

iii) De 1990 à 2014 : déploiement du système d'information génétique collectif, mise en place des évaluations IBOVAL, déploiement du testage des qualités maternelles en ferme en race Charolaise, valorisation des données post-sevrage et d'abattage.

iv) Depuis 2015, mise en place de la sélection génomique pour les races Charolaise, Limousine et Blonde d'Aquitaine.

est présentée en partant de la naissance des veaux. Cette description détaillée est résumée sur la figure 1 .

\section{1 / Autour de la naissance}

L'éleveur est le premier maillon de l'identification pérenne généralisée et de la traçabilité des bovins. Il notifie ses naissances en renseignant le numéro de la boucle apposée sur le veau, la date de naissance, le sexe, le type racial (et celui des parents), le nom éventuel, le numéro de la mère ayant vêlé et le numéro du père.

Dans le cas où cet éleveur est adhérent au service CPB, les parentés sont certifiées en utilisant des règles de gestion et un code race est attribué. Cette certification des parentés est incontournable pour valoriser ces informations en sélection. En outre, l'éleveur notifie des informations complémentaires (Institut de l'Élevage 2008) à la fin de chaque gestation :

i) l'origine de la gestation (transplantation embryonnaire ou gestation classique);

ii) la fin de gestation (avortement ou à terme) ;

iii) la naissance gémellaire ou unique ;

iv) la note de conditions de naissance (de 1 à 5);

v) le poids de naissance du veau en $\mathrm{kg}$;

Figure 1. Schéma de collecte des performances en bovins allaitants au cours de la vie des animaux dans différents outils (références 2015).

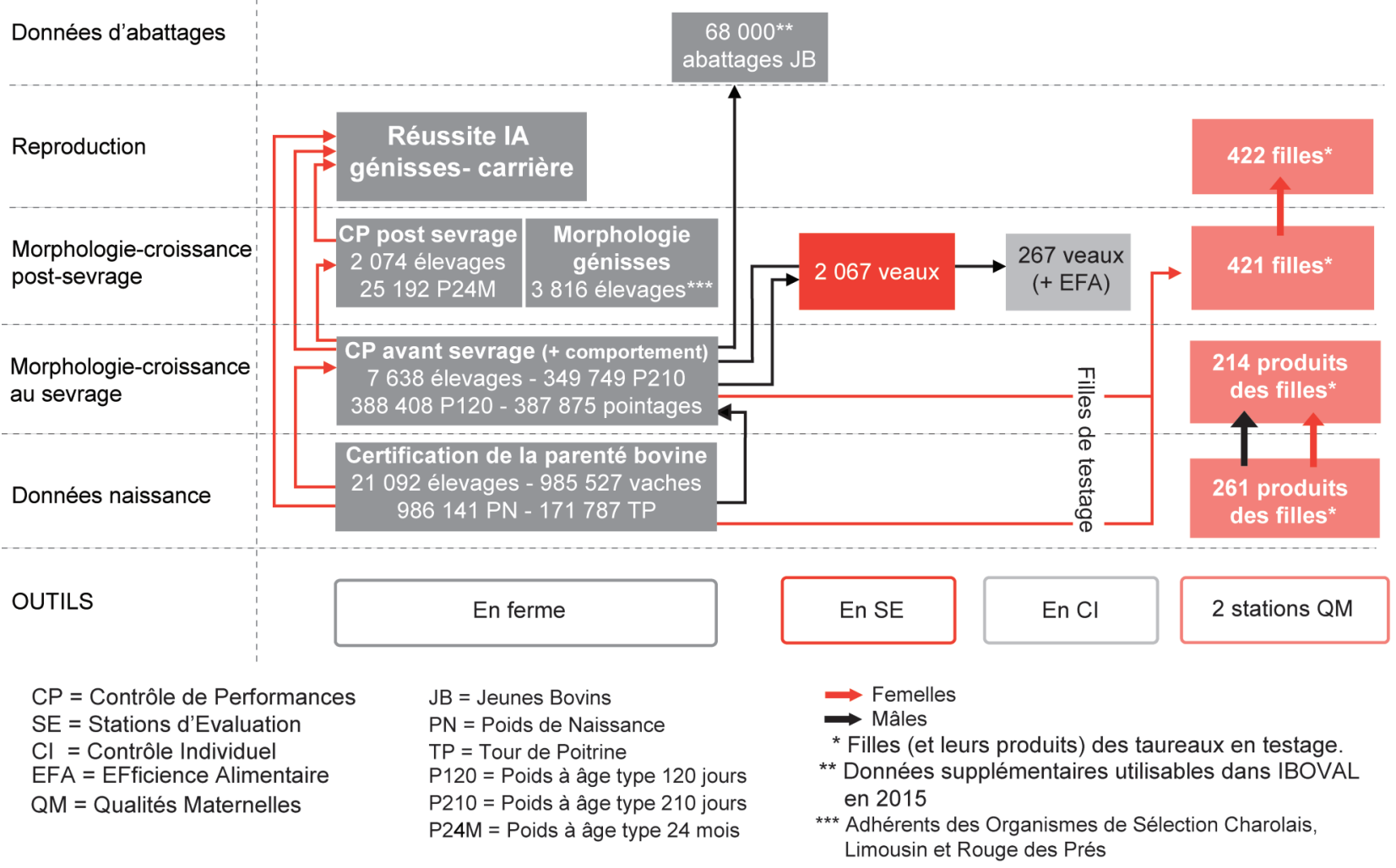


vi) depuis 2013, le mode d'obtention de ce poids (pesé ou non pesé) et le tour de poitrine en $\mathrm{cm}$ (Institut de l'Élevage 2013).

\section{2 / Jusqu'au sevrage}

Ensuite, pour les élevages adhérents au contrôle de performances (protocole avec des pesées VA4), les veaux sont contrôlés jusqu'au sevrage. Dans ce cas, tous les veaux présents de moins de 301 jours d'âge (règle d'exhaustivité) doivent être pesés au moins deux fois de façon à obtenir un poids à âge type (poids à un âge donné observé ou calculé à partir d'autres poids relevés à des dates proches) à 120 jours (P120) et/ou à 210 jours (P210). Les pesées doivent être espacées d'au moins 60 jours et d'au plus 210 jours. Dans le cas où l'âge type est en dehors de l'intervalle entre les deux pesées, il ne doit pas être éloigné de plus de 30 jours de la pesée la plus proche. Les pesées sont réalisées soit par l'organisme « Bovins Croissance », soit par l'éleveur lui-même dans le cadre d'un contrat spécifique. Les poids de vente en vif sont collectés selon des règles spécifiques. La morphologie des animaux est également décrite à un âge compris entre 5 et 10 mois ( 150 à 300 jours) par un technicien autorisé par la commission en charge des agréments de France Génétique Élevage. Cette description (appelée pointage) comprend dix-neuf postes notés de 1 à 10. Ces postes sont regroupés en trois notes composites exprimées sur 100 points (Institut de l'Élevage 2014) : i) Développement Musculaire (DM), ii) Développement Squelettique (DS) et iii) Aptitudes Fonctionnelles (AF). Pour documenter les performances, le technicien a la possibilité de renseigner des groupes de conduite et des situations individuelles particulières (ex. préparation à un concours), identifiant des conduites différentes au sein d'un troupeau. Enfin, depuis peu, les organismes « Bovins Croissance " peuvent évaluer le comportement du veau à la pesée (comptage de 1 à 10 et plus du nombre de mouvements dans la cage durant 10 secondes), ainsi qu'au pointage (note de 1 à 7) (Venot et al 2015).

\section{3 / Au-delà du sevrage}

\section{a) Pour les mâles en station}

Les mâles recrutés en Stations d'Évaluation (SE) et les candidats à l'Insémination Animale (IA) passant par une station de Contrôle Individuel (CI), âgés de 7 à 12 mois, sont contrôlés sur leur morphologie et sur leur croissance. Pour les jeunes taureaux en CI, un contrôle de leur efficacité alimentaire est également réalisé en mesurant les quantités ingérées d'un aliment spécifique, grâce à des distributeurs automatiques de concentrés. Cet aliment condensé dans un bouchon intégrant des fibres, de valeur énergétique allant de 0,7 à $0,8 \mathrm{UFV} / \mathrm{kg}$ de matière sèche (unités fourragères viande), est distribué ad libitum. Ce contrôle de l'efficacité alimentaire effectué en station de CI mais pas en SE constitue la principale différence de protocole entre ces outils. Dans les deux cas, pour rendre plus objective la hiérarchisation des reproducteurs, le protocole impose de :

i) disposer d'au moins 36 animaux dans une série composée de groupes de contemporains de 12 animaux au minimum avec des écarts d'âge intra groupe de 42 jours au maximum;

ii) avoir une durée d'adaptation d'au moins 28 jours pour habituer les mâles à l'aliment et à leur nouvel environnement, démarrant par une pesée simple à l'entrée ;

iii) avoir une durée de contrôle de 84 jours pour mesurer la croissance grâce à une double pesée en début et en fin de période ; un suivi de la croissance par une pesée simple tous les 28 jours complète le dispositif;

iv) décrire la morphologie des animaux (notes composites DM, DS, AF et qualités de race, dont la construction est spécifique à chacune des races) et réaliser des mensurations externes dans les semaines qui précèdent ou qui suivent la fin de la période de contrôle de croissance ;

v) réaliser des mesures de mensurations internes (ouverture pelvienne) dans les jours qui suivent la fin de la période de contrôle de croissance.

En SE, un objectif minimum de croissance est défini par race afin d'assurer une bonne expression du potentiel de croissance : $1400 \mathrm{~g} / \mathrm{j}$ en race Charolaise ; $1200 \mathrm{~g} / \mathrm{j}$ en Limousine ; $1300 \mathrm{~g} / \mathrm{j}$ en Blonde d'Aquitaine ; $1300 \mathrm{~g} / \mathrm{j}$ en Salers ; $1100 \mathrm{~g} / \mathrm{j}$ en Aubrac; $1200 \mathrm{~g} / \mathrm{j}$ en Gasconne; $1200 \mathrm{~g} / \mathrm{j}$ en Parthenaise. En station de CI, il n'y a pas d'objectif de croissance mais un pilotage fin des quantités journalières distribuées permet d'atteindre un niveau d'alimentation ad libitum.

\section{b) Pour les femelles en ferme}

En plus des pesées au sevrage de leurs veaux, les élevages adhérents à un organisme «Bovins Croissance» peuvent choisir de réaliser sur leurs génisses des pesées post-sevrage (contrat VPS, pour vaches allaitantes post-sevrage). L'objectif est de mesurer la croissance post-sevrage des génisses par l'intermédiaire des poids à âge type à 12 mois (P12M), 18 mois (P18M) et 24 mois (P24M). Les éleveurs volontaires s'engagent pour cela à peser (contrat éleveur) ou à faire peser toutes leurs génisses âgées de 10 à 28 mois n'ayant pas vêlé. Afin de calculer a minima un P18M ou un P24M, trois pesées doivent être réalisées, avec un intervalle d'au moins 60 jours et d'au plus 300 jours entre deux pesées. Dans le cas où l'âge type est en dehors de l'intervalle entre deux pesées, il ne doit pas être éloigné de plus de 30 jours de la pesée la plus proche.

Pour les éleveurs adhérant au service élaboré de l'Organisme de Sélection de leur race (6\% des élevages), une collecte de la morphologie post-sevrage des génisses est organisée selon la race entre 18 mois (premier vêlage à 24 mois) et 42 mois (Charolaise, Limousine, Rouge des Prés et Salers, en cours de déploiement pour la Blonde d'Aquitaine). La liste des postes pointés dans chaque race peut légèrement varier en fonction des priorités raciales. Cependant, le socle est commun avec les pointages réalisés au sevrage (Institut de l'Élevage et INRA 2014). Certaines notes composites sont communes entre les races (DS, DM et AF voir glossaire), alors que d'autres sont spécifiques à certains types génétiques: les qualités de race en Rouge des Prés, le bassin en Limousine et la conformité au standard en Charolaise (notée ---, --, -, $=,+,++$ et +++ et retransformée pour l'évaluation en valeur numérique).

En ce qui concerne la reproduction, deux nouveaux critères ont été déployés début 2014. D'une part, un critère de réussite à l'IA première est calculé pour toute génisse et toute vache primipare inséminées, à partir des IA réalisées et des résultats de mise bas enregistrés dans les élevages à la $\mathrm{CPB}$, pour évaluer l'aptitude des génisses à être fécondées à la première IA et à conduire cette gestation à son terme. D'autre part, un critère mesurant l'efficacité de carrière (exprimé en nombre de veaux nés, produits par la vache) est calculé pour toute vache du troupeau, quel que soit son âge. Ce critère d'efficacité est défini comme le nombre de veaux nés par femelle jusqu'à l'âge de 6,5 ans.

c) Mesures sur les femelles conduites en station de contrôle sur descendance

L'objectif du testage sur descendance des taureaux est de rassembler en station, afin de les conduire dans des conditions de milieu similaires, les filles d'au moins 6 taureaux, pour mesurer :

i) leur croissance et leur morphologie post-sevrage ;

ii) leur fertilité ;

iii) leur facilité de vêlage ;

iv) leur aptitude à l'allaitement. 
Généralement, les séries sont composées d'au moins 10 taureaux au moment de la mise en place des IA et d'un témoin permettant les connexions entre les séries. Une élimination des descendances se fait au fur et à mesure de l'arrivée de résultats. Au final, 6 à 9 descendances terminent le protocole.

Pour cela, des filles procréées en ferme sont regroupées au sevrage dans les stations de Moussours-Uzerche (Corrèze) pour la race Limousine et de Casteljaloux (Lot-et-Garonne) pour la race Blonde d'Aquitaine. Il faut disposer d'au moins 20 filles par taureau pour évaluer la croissance/morphologie et la fertilité, et d'au moins 12 filles qui vêlent et 10 veaux sevrés pour évaluer la facilité de vêlage et l'aptitude à l'allaitement, respectivement.

Les mesures ou observations effectuées sur les femelles sont :

i) des pesées mensuelles avant vêlage pour établir un P18M ;

ii) des mensurations externes ainsi qu'une description morphologique réalisées à 18 mois pour calculer le Développement Musculaire (DM), le Développement Squelettique (DS), les Aptitudes Fonctionnelles (AF) (cf. glossaire) et les qualités de race ;

iii) trois informations de fertilité : la puberté (observation ou non d'un œstrus avant 15 mois), la fertilité (réussite ou non aux IA sur chaleurs naturelles au cours d'une période de 70 jours) et la viabilité des veaux au sevrage (veaux vivants au sevrage par rapport aux nés) ;

iv) pour le vêlage qui a lieu à 2 ans, les observations de préparation au vêlage, le poids de naissance et la note de conditions de naissance des veaux (de 1 à 5 comme à la $\mathrm{CPB}$ ), le poids au vêlage des mères et leur ouverture pelvienne (au plus tard dans les 10 jours qui suivent le vêlage);

v) pour l'aptitude à l'allaitement, les poids des veaux : pesées mensuelles afin de calculer des poids à âge type 120 et 180 jours. La production laitière est également mesurée à 60 et 120 jours, par pesées des veaux avant et après la tétée (matin et soir), après une séparation de 12 heures du couple mère-veaux. La morphologie des veaux est également décrite au sevrage (comme en ferme).

\section{4 / À l'abattage des animaux}

Enfin, NORMABEV, l'association technique interprofessionnelle du bétail et des viandes créée en 2002 par INTERBEV (Interprofession Bétail et Viande), chargée entre autres de l'organisation de la circulation des informations d'abattage, met à disposition de l'Institut de l'Elevage, deux fois par an, l'ensemble des données d'abattage collectées depuis 2006. Ces informations comprenant le poids de carcasse, la date d'abattage qui permet de déduire l'âge à l'abattage, la note EUROP de conformation (FranceAgriMer 2016) et la note de couleur de la viande (pour les veaux seulement), sont valorisées dans les évaluations IBOVAL. Les données utilisées sont celles de jeunes bovins de race pure et de veaux de boucherie croisés (Holstein $\mathrm{x}$ races à viande ou Montbéliarde $\mathrm{x}$ Charolais).

Le tableau 1 indique le nombre de données collectées annuellement dans ces différents outils. Le taux de pénétration de la collecte de performances (vaches concernées par rapport au total des vaches) est de $26 \%$ pour l'ensemble des races. Il varie de $23 \%$ en Limousine à $58 \%$ en Bazadaise. La collecte est plus développée dans les races à plus faibles effectifs. Les races Charolaise et Limousine représentent une grande part des effectifs contrôlés.

Le contrôle de performances avec pesées représente $35 \%$ des veaux pesés à la naissance (nombre de phénotypes pour P210 divisé par le nombre de poids de naissance enregistrés). Le taux de veaux pesés à la naissance ayant un phénotype pour P210 est plus faible chez les mâles pour lesquels la mortalité est plus forte. Les races Charolaise, Limousine, Parthenaise et Salers ont une pénétration du contrôle de performances avec pesées plus forte que les autres races (taux de femelles pesées à la naissance ayant un phénotype pour P210 supérieur à $40 \%$ ). Le contrôle de performances avec pesées est moins développé en Aubrac, en Gasconne et en Blonde d'Aquitaine.

Il y a généralement plus de veaux pointés au sevrage que de veaux pour lesquels un poids à âge type à 210 jours est calculé. Les règles pour l'obtention des poids à âge type sont plus contraignantes et certains veaux quittent les élevages trop jeunes pour disposer d'un phénotype pour P210.

Les pesées post-sevrage sont surtout développées en race Charolaise $(82 \%$ du total des génisses ayant un phénotype pour $\mathrm{P} 24 \mathrm{M}$ ).

Au niveau des stations, c'est la race Limousine qui présente les effectifs les plus importants. La race Rouge des Prés ne dispose que d'une station de CI, les races rustiques n'ayant que des SE. Enfin, les effectifs d'animaux en contrôle sur descendance sont du même ordre dans les races Limousine et Blonde d'Aquitaine.
Les données collectées à travers l'ensemble des outils décrits précédemment sont ensuite utilisées pour les valoriser lors des évaluations génétiques afin de calculer les « index » des reproducteurs et des jeunes candidats, et de permettre aux éleveurs de prendre leurs décisions de sélection et de réforme.

\section{2 / Estimation des valeurs génétiques dans les popula- tions bovines allaitantes}

Dans une population d'animaux d'élevage, après chaque cycle de reproduction, d'anciens reproducteurs sont réformés et remplacés par de jeunes individus. La sélection consiste donc à choisir de façon orientée les reproducteurs de renouvellement parmi les candidats, ainsi que les animaux à réformer, sur la base de leur valeur génétique. De ces choix répétés de génération en génération, on attend une élévation progressive du niveau génétique de la population pour les caractères inclus dans l'objectif de sélection.

Pour réaliser cette sélection, les éleveurs et les entreprises de sélection s'appuient sur des « index », qui sont des prédictions (ou estimations, ces deux termes étant ici employés de manière équivalente) de la valeur génétique des animaux. Ces index sont obtenus à partir des phénotypes des animaux, de leur généalogie, et dans certains cas de leurs génotypes.

Le génome bovin est constitué de 30 paires de chromosomes sur lesquels se trouvent des régions appelées QTL («Quantitative Trait Loci ») ayant un effet sur la variabilité des phénotypes. Le terme QTL reflète un effet statistique dont on ne connait généralement pas l'origine biologique : il peut s'agir d'une ou plusieurs mutations localisées dans un ou plusieurs gènes ou dans des régions intergéniques. Ces effets peuvent être favorables ou défavorables selon la forme (l'allèle) du QTL portée par l'animal. Selon les allèles aux QTL qu'il porte sur son génome, un individu aura donc une valeur génétique favorable ou défavorable, pour un caractère donné, par rapport au reste de la population. L'objectif des évaluations génétiques est d'estimer cette valeur génétique au travers de différents modèles présentés ci-dessous.

\section{1 / Évaluations génétiques poly- géniques}

Depuis les années 1990 (à l'exception des évaluations génomiques mises en place en 2015 qui seront décrites dans la partie suivante), toutes les évaluations 
Tableau 1. Effectifs d'animaux contrôlés en ferme et en station en France (source bilan de contrôle de performances 2015, bilans SE-Cl et bilans contrôle sur descendance).

\begin{tabular}{|c|c|c|c|c|c|c|c|c|c|c|c|c|}
\hline \multirow[b]{2}{*}{ Animaux } & \multirow[b]{2}{*}{ Performances } & \multicolumn{11}{|c|}{ Référence 2015 * } \\
\hline & & $\begin{array}{l}\text { Toutes } \\
\text { races }\end{array}$ & AUB & BAZ & BLA & CHA & GAS & INRA & LIM & PAR & ROU & SAL \\
\hline \multicolumn{13}{|c|}{ Certification de la parenté bovine et contrôle de performances en ferme } \\
\hline \multicolumn{2}{|c|}{$\begin{array}{l}\% \text { de vaches concernées } \\
\text { par rapport à la population } \\
\text { totale }\end{array}$} & 26 & 38 & 58 & 30 & 24 & 44 & & 23 & 50 & 46 & 25 \\
\hline \multirow{8}{*}{ Femelles } & PN & 484799 & 27445 & 952 & 77197 & 184821 & 3502 & & 125421 & 11562 & 9044 & 18052 \\
\hline & TP & 84307 & 4001 & & 15845 & 36180 & & & 19243 & & 2171 & \\
\hline & P120 & 194960 & 7017 & 342 & 21467 & 89414 & 874 & & 57891 & 7132 & 3276 & 8231 \\
\hline & P210 & 179389 & 6654 & 278 & 17516 & 84625 & 731 & & 53159 & 6825 & 2928 & 7285 \\
\hline & $\begin{array}{l}\text { Pointées au } \\
\text { sevrage }\end{array}$ & 198244 & 7233 & 276 & 21787 & 92728 & 986 & & 57442 & 7074 & 3257 & 8357 \\
\hline & P12M & 62681 & 478 & 163 & 6085 & 39279 & 168 & & 12415 & 2707 & 1076 & 1008 \\
\hline & P18M & 37186 & 120 & 109 & 3278 & 27215 & 40 & & 4854 & 1163 & 613 & 423 \\
\hline & P24M & 25192 & 46 & 69 & 1452 & 20607 & 17 & & 2216 & 633 & 401 & 222 \\
\hline \multirow{5}{*}{ Mâles } & PN & 501342 & 28776 & 934 & 78663 & 192449 & 3499 & & 128994 & 11748 & 9494 & 18507 \\
\hline & TP & 87480 & 4239 & & 16229 & 37439 & & & 20228 & & 2220 & \\
\hline & P120 & 193448 & 7331 & 309 & 19536 & 89975 & 835 & & 57166 & 6910 & 3367 & 8465 \\
\hline & P210 & 170360 & 6900 & 192 & 12979 & 83351 & 687 & & 50195 & 6191 & 2703 & 7473 \\
\hline & $\begin{array}{l}\text { Pointés au } \\
\text { sevrage }\end{array}$ & 189631 & 7495 & 349 & 18761 & 90057 & 823 & & 54672 & 6601 & 3292 & 8292 \\
\hline \multicolumn{13}{|c|}{ Stations de Contrôle Individuel (Cl) et Stations d'Evaluation (SE) } \\
\hline \multirow{2}{*}{$\begin{array}{l}\text { Jeunes } \\
\text { mâles }\end{array}$} & Contrôlés Cl & 273 & & & 46 & 111 & & 10 & 44 & & 72 & \\
\hline & Contrôlés SE & 2091 & 129 & 7 & 214 & 654 & 81 & & 828 & 93 & & 92 \\
\hline \multicolumn{13}{|c|}{ Contrôle sur descendance en station } \\
\hline \multirow{3}{*}{$\begin{array}{l}\text { Filles de } \\
\text { testage }\end{array}$} & P18M & 421 & & & 211 & & & & 210 & & & \\
\hline & $\begin{array}{l}\text { Pointées à } 18 \\
\text { mois }\end{array}$ & 421 & & & 211 & & & & 210 & & & \\
\hline & $\begin{array}{l}\text { Mises à la } \\
\text { reproduction }\end{array}$ & 422 & & & 212 & & & & 210 & & & \\
\hline \multirow{4}{*}{ Veaux } & Nés utiles & 261 & & & 135 & & & & 126 & & & \\
\hline & P120 & 215 & & & 113 & & & & 102 & & & \\
\hline & \begin{tabular}{|l|} 
Pesés pour \\
contrôle laitier
\end{tabular} & 218 & & & 113 & & & & 105 & & & \\
\hline & Pointés & 214 & & & 113 & & & & 101 & & & \\
\hline
\end{tabular}

AUB $=$ AUBrac $; \mathrm{BAZ}=\mathrm{BAZadaise} ; \mathrm{BLA}=$ BLonde d'Aquitaine $; \mathrm{CHA}=\mathrm{CHArolaise} ; \mathrm{GAS}=\mathrm{GASconne} ;$ INRA $=$ INRA $95 ;$ LIM $=$ LIMousine $;$ PAR = PARthenaise $;$ ROU = ROUge des Prés $;$ SAL = SALers $; \mathrm{PN}=$ Poids Naissance $; \mathrm{TP}=$ Tour de Poitrine $;$ P120 = Poids-Age Type (PAT) 120 jours ; P210 = PAT 210 jours ; P12M = PAT 12 mois ; P18M = PAT 18 mois ; P24M = PAT 24 mois.

Certification de la parenté bovine et contrôle de performances :

${ }^{*}$ Référence $2015 \rightarrow$ animaux nés du 01/08/14 au 31/07/15 pour les performances avant sevrage et au sevrage.

$\rightarrow$ animaux nés du 01/08/11 au 31/07/12 pour les données post-sevrage (P12M, P18M, P24M).

Contrôles individuel et stations d'évaluation :

${ }^{*}$ Référence $2015 \rightarrow$ animaux contrôlés au deuxième semestre 2014 et au premier semestre 2015.

Contrôlés en $\mathrm{Cl}$ : croissance - morphologie, ouverture pelvienne, mensurations, efficience alimentaire.

Contrôlés en SE : croissance - morphologie, ouverture pelvienne, mensurations.

Contrôle sur descendance en station :

${ }^{*}$ Référence $2015 \rightarrow$ filles et produits des filles des taureaux évalués « qualités maternelles » en 2015

génétiques officielles réalisées dans les populations bovines allaitantes françaises s'appuient sur le « modèle génétique infinitésimal » (également appelé « modèle polygénique »), qui considère le génome comme une « boîte noire » contenant un très grand nombre de QTL non identifiés ayant chacun un effet faible sur les carac- tères. Le principe sous-jacent à ce modèle est qu'un animal ayant un bon phénotype pour un caractère d'intérêt est majoritairement porteur d'allèles favorables aux QTL impliqués dans le déterminisme de ce caractère. S'il est mis à la reproduction, cet animal transmettra aléatoirement à ses descendants la moitié de ses allè- les, et donc une partie de sa supériorité génétique. Les animaux apparentés d'une population ont donc une partie de leurs allèles aux QTL identiques parce qu'ils les ont reçus d'un ancêtre commun. En conséquence, leurs valeurs génétiques se ressemblent d'autant plus qu'ils sont apparentés. 
Sous ce modèle polygénique, la valeur génétique des individus va être prédite en utilisant la méthode statistique du BLUP («Best Linear Unbiased Prediction », Henderson 1975) appliquée à un modèle génétique individuel (Quaas et Pollack 1980) appelé couramment « modèle animal » à partir des phénotypes et des généalogies enregistrées dans la population, et ceci sans chercher à identifier les QTL responsables des différences de potentiel génétique entre animaux. La sélection des allèles favorables aux QTL dans la population se fait ainsi implicitement.

\section{a) Cas d'un caractère uniquement soumis à un effet génétique direct}

Dans le cas le plus simple, le phénotype d'un animal dépend, d'une part, de sa valeur génétique résultant de l'effet des allèles aux QTL qu'il porte, et d'autre part, des effets combinés du milieu dans lequel il a été élevé et a exprimé son phénotype. Sous le modèle animal, la performance d'un individu est décomposée de la manière suivante :

$$
y_{i}=\Sigma_{j} m_{j}+a_{i}+e_{i} \quad \text { (1) où : }
$$

i) $\mathrm{y}_{\mathrm{i}}$ est le phénotype de l'animal i ;

ii) $\sum_{j} \mathrm{~m}_{\mathrm{j}}$ est la somme des effets de milieu j identifiés ayant un impact sur le phénotype, tels que l'élevage, l'année de naissance, le rang de vêlage de sa mère, l'âge de l'animal au moment de la mesure, son sexe etc ;

iii) $\mathrm{a}_{\mathrm{i}}$ est la valeur génétique additive directe de l'animal i, égale à la somme des effets des allèles aux QTL affectant le phénotype portés par l'animal ; c'est le double de ce qu'il transmettra en moyenne à ses descendants ;

iv) $\mathrm{e}_{\mathrm{i}}$ est la résiduelle du modèle, qui englobe tous les effets de tous les facteurs non explicitement inclus dans le modèle : effets génétiques non additifs (interactions entre allèles d'un QTL et entre QTL), effets environnementaux non identifiés ou non enregistrables, erreurs de mesure de la performance, etc.

Pour plus de détails sur le modèle individuel et les hypothèses associées aux différents effets, voir par exemple Ducrocq (1992). L'utilisation de ce type de modèle suppose la connaissance préalable de l'héritabilité du caractère, c'est-à-dire de la part (entre 0 et 1 ) de la variabilité totale des phénotypes qui est d'origine génétique additive. Plus l'héritabilité est élevée, plus la part des variations phénotypiques expliquée par la composante génétique est importante, et donc plus les différences observées entre les phénotypes des reproducteurs sont transmissibles à leurs descendants.
La méthode du BLUP appliquée au modèle animal permet ainsi de tirer profit des performances de l'individu si elles ont été mesurées, et également des performances de tous ses apparentés. Les effets de milieu et les valeurs génétiques additives de l'ensemble des animaux étant estimés simultanément, les estimations de ces dernières sont en théorie comparables entre elles, quel que soit l'âge des animaux ou le milieu dans lequel ils ont été élevés.

La précision de la valeur génétique estimée de chaque individu dépend du nombre de mesures de performances disponibles pour réaliser cette estimation (performance propre de l'individu et des apparentés) et du nombre d'animaux contrôlés présents dans les différentes conditions de milieu, permettant une plus ou moins bonne correction des performances pour les effets environnementaux considérés dans le modèle. La précision d'estimation des valeurs génétiques additives est exprimée au travers du Coefficient de Détermination (CD) qui accompagne la publication des index et qui varie entre 0 (précision nulle) et 1 (précision maximum).

Dans le cas particulier des caractères de qualités maternelles mesurés en station de testage sur descendance, le modèle génétique utilisé est un modèle " père ", dans lequel les performances des filles d'un taureau sont exprimées par rapport à la valeur génétique de leur père (qui est celle qu'on estime).

\section{b) Cas d'un caractère soumis à des effets} génétiques directs et maternels

Pour certains caractères, la performance d'un individu est la résultante, non seulement de l'effet des allèles aux QTL portés par l'animal (c'est-à-dire de son effet génétique direct) et de l'effet de son environnement, mais également de facteurs maternels, comme l'environnement utérin ou la production laitière de sa mère. Ces caractères s'expriment en général tôt dans la vie de l'animal, lorsqu'ils dépendent le plus de leur mère, comme le poids et les conditions de naissance, la croissance jusqu'au sevrage, etc.

Les effets maternels incluent généralement une composante génétique résultant des allèles aux QTL portés par la mère (effet génétique additif maternel) et une composante dite «environnementale » non transmissible qui s'exerce sur tous les veaux de la mère (regroupant les effets génétiques maternels non additifs et les effets d'environnement permanent non génétique lié à la mère). Dans ce cas, l'équation (1) décrivant la performance de l'individu i de mère $\mathrm{k}$ dans le milieu j devient :

$$
y_{i k}=\sum_{j} m_{j}+a_{i k}+d_{k}+p_{k}+e_{i k}(2) \text { où : }
$$

i) $\mathrm{a}_{\mathrm{ik}}$ est la valeur génétique additive directe de l'animal $\mathrm{i}$ issu de la mère $\mathrm{k}$;

ii) $\mathrm{d}_{\mathrm{k}}$ est la valeur génétique additive maternelle de la mère $\mathrm{k}$;

iii) $\mathrm{p}_{\mathrm{k}}$ est l'effet d'environnement permanent maternel de la mère $\mathrm{k}$;

iv) $\mathrm{e}_{\mathrm{ik}}$ est la résiduelle du modèle, qui englobe les effets de tous les facteurs non inclus dans le modèle.

On peut illustrer ce modèle en considérant l'exemple de la vitesse de croissance jusqu'au sevrage d'un veau dont la mère aurait été mise à la reproduction précocement. Ce facteur environnemental défavorable au développement de sa mamelle l'empêche d'exprimer pleinement son potentiel génétique laitier. Dans ce cas, le phénotype du veau va effectivement dépendre à la fois de son propre environnement (m), de sa propre valeur génétique (directe) pour la croissance (a) et d'un effet maternel, qui est lui-même le résultat de la valeur génétique laitière de la mère $(\mathrm{d})$ qui ne peut pas s'exprimer ici totalement en raison d'un problème de conduite d'élevage (p) qui affecte la croissance du veau (et qui affectera de la même manière la croissance de tous les autres veaux de cette mère).

Dans le cas d'un caractère soumis à des effets maternels, on estime donc pour chaque individu de la population et quel que soit son sexe, à la fois son effet génétique additif direct (a) et son effet génétique additif maternel (d). Mais seules les vaches exprimeront l'effet génétique maternel : en moyenne, l'apport d'un veau femelle aux performances de ses potentiels produits sera $0,5 \times \mathrm{a}+\mathrm{d}$, alors que celui d'un veau mâle se limitera à $0,5 \times$ a. En revanche, tous les animaux (les taureaux comme les vaches) transmettent à leurs veaux en moyenne la moitié de leur valeur génétique additive directe et la moitié de leur valeur génétique additive maternelle : il est donc important de tenir compte de la valeur de l'effet génétique maternel également lors de la sélection des mâles.

\section{c) Évaluations génétiques multi-caractères}

Certains caractères ont une variabilité qui dépend en partie des mêmes QTL. Par exemple, on peut imaginer que le poids à la naissance d'un animal et son poids au sevrage sont contrôlés en partie par les mêmes QTL. De tels caractères sont génétiquement corrélés et ils peuvent alors être évalués conjointement (voir tableau 2). La réalisation d'évaluations génétiques multi-caractères permet d'augmenter la précision de l'estimation des valeurs génétiques, puisque les phénotypes pour un caractère 
donné apportent une information sur les effets génétiques pour les caractères qui lui sont génétiquement corrélés. Ce gain de précision est d'autant plus important que la corrélation génétique entre les caractères est estimée de façon fiable, qu'elle est élevée, et que les caractères associés au caractère considéré ont des héritabilités élevées.

Tableau 2. Inventaire des principaux caractères évalués et évaluations génétiques réalisées dans les principales populations allaitantes françaises (caractères mesurés en ferme ou en abattoirs, évaluation IBOVAL).

\begin{tabular}{|c|c|c|c|c|c|c|c|c|c|c|c|c|c|c|c|}
\hline \multirow{2}{*}{ Aptitude } & \multirow{2}{*}{ Caractère } & \multicolumn{9}{|c|}{ Race } & \multirow{2}{*}{$\begin{array}{c}\text { Modèle } \\
\text { multi- } \\
\text { caractère* }\end{array}$} & \multirow{2}{*}{ Envp** } & \multicolumn{2}{|c|}{$\begin{array}{c}\text { Héritabilité } \\
\text { (Moyenne [Minimum-Maximum]) }\end{array}$} & \multirow{2}{*}{$\begin{array}{c}\text { Index } \\
\text { élémentaire } \\
\text { (Direct/Maternel) }\end{array}$} \\
\hline & & 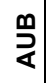 & $\underset{\substack{N \\
\Phi}}{ }$ & 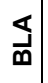 & $\stackrel{\mathbb{1}}{\mathrm{U}}$ & đ্ & $\leqq$ & $\frac{\alpha}{\alpha}$ & ठे & હ & & & directe & maternelle & \\
\hline \multirow{2}{*}{ Naissance } & PN & $\mathrm{x}$ & $x$ & $x$ & $\mathrm{x}$ & $x$ & $x$ & $x$ & $x$ & $x$ & A & $x$ & $0,39[0,15-0,56]$ & $0,08[0,04-0,14]$ & \\
\hline & $\mathrm{CN}$ & $\mathrm{x}$ & $x$ & $x$ & $\mathrm{x}$ & $x$ & $x$ & $x$ & $x$ & $x$ & A & $x$ & $0,11[0,05-0,19]$ & $0,04[0,01-0,06]$ & $-/$ AVel \\
\hline \multirow{2}{*}{$\begin{array}{l}\text { Croissance } \\
\text { sevrage }\end{array}$} & P120 & $x$ & $x$ & $x$ & & $x$ & $x$ & $x$ & $x$ & $x$ & B & $x$ & $0,24[0,17-0,30]$ & $0,12[0,09-0,21]$ & \\
\hline & P210 & $x$ & $\mathrm{x}$ & $x$ & $x$ & $x$ & $x$ & $x$ & $x$ & $x$ & B & $\mathrm{x}$ & $0,24[0,16-0,36]$ & $0,19[0,07-0,19]$ & CRsev / ALait \\
\hline \multirow{3}{*}{$\begin{array}{l}\text { Morphologie } \\
\text { sevrage }\end{array}$} & DM & $x$ & $x$ & $x$ & $\mathrm{x}$ & $x$ & $x$ & $x$ & $x$ & $\mathrm{x}$ & C & $x$ & $0,29[0,18-0,53]$ & & DMsev \\
\hline & DS & $x$ & $x$ & $x$ & $\mathrm{x}$ & $x$ & $x$ & $x$ & $x$ & $x$ & C & $x$ & $0,23[0,11-0,33]$ & & DSsev \\
\hline & GC & $x$ & $x$ & $x$ & $x$ & $x$ & $x$ & $\mathrm{x}$ & $x$ & $x$ & C & $\mathrm{x}$ & $0,17[0,13-0,21]$ & & FOSsev \\
\hline \multirow{2}{*}{$\begin{array}{l}\text { Compor- } \\
\text { tement }\end{array}$} & REAC & $x$ & $x$ & $x$ & $x$ & $x$ & $x$ & $x$ & $x$ & $x$ & non & $x$ & $0,15[0,12-0,17]$ & & REACsev \\
\hline & COMP & $\mathrm{x}$ & $x$ & $x$ & $x$ & $x$ & $x$ & $x$ & $x$ & $x$ & non & $x$ & $0,10[0,09-0,11]$ & & COMPsev \\
\hline \multirow{3}{*}{ Vie productive } & RIAPG & $x$ & & $x$ & $x$ & & $x$ & $x$ & $x$ & $x$ & $\mathrm{D}$ & & 0,015 & & RIAPgef \\
\hline & RIAPP & $x$ & & $x$ & $\mathrm{x}$ & & $x$ & $x$ & $x$ & $x$ & D & & 0,015 & & \\
\hline & EFCAR & $\mathrm{x}$ & $x$ & $\mathrm{x}$ & $\mathrm{x}$ & $x$ & $x$ & $x$ & $x$ & $x$ & $\mathrm{E}$ & & 0,04 & & EFCAR \\
\hline \multirow{5}{*}{$\begin{array}{l}\text { Croissance } \\
\text { post-sevrage }\end{array}$} & P120 & & $x$ & $\mathrm{x}$ & & & $x$ & $x$ & $\mathrm{x}$ & $x$ & $\mathrm{~F}$ & $x$ & $0,32[0,26-0,34]$ & & \\
\hline & P210 & & $x$ & $\mathrm{x}$ & $x$ & & $x$ & $x$ & $x$ & $x$ & $\mathrm{~F}$ & $\mathrm{x}$ & $0,34[0,22-0,37]$ & & \\
\hline & P12M & & $x$ & $x$ & $x$ & & $x$ & $x$ & $\mathrm{x}$ & $x$ & $F$ & & $0,40[0,32-0,42]$ & & \\
\hline & P18M & & $\mathrm{x}$ & $x$ & $x$ & & $x$ & $x$ & $\mathrm{x}$ & $x$ & $\mathrm{~F}$ & & $0,42[0,34-0,45]$ & & \\
\hline & $\mathrm{P} 24 \mathrm{M}$ & & $x$ & $x$ & $x$ & & $x$ & $x$ & $x$ & $x$ & $\mathrm{~F}$ & & $0,43[0,24-0,47]$ & & CRpsf \\
\hline \multirow{6}{*}{$\begin{array}{l}\text { Morphologie } \\
\text { post-sevrage }\end{array}$} & DM & & & & $x$ & & $x$ & & $x$ & & G & $x$ & $0,32[0,32-0,32]$ & & \\
\hline & DS & & & & $x$ & & $x$ & & $x$ & & G & $\mathrm{x}$ & $0,27[0,25-0,30]$ & & \\
\hline & DM30M & & & & $x$ & & $\mathrm{x}$ & & $x$ & & G & & $0,34[0,28-0,37]$ & & DMpsf \\
\hline & DS30M & & & & $x$ & & $x$ & & $x$ & & G & & $0,43[0,42-0,43]$ & & DSpsf \\
\hline & AF30M & & & & $x$ & & $x$ & & $x$ & & G & & $0,18[0,14-0,25]$ & & AFpsf \\
\hline & GC30M & & & & $x$ & & $x$ & & $x$ & & G & & $0,31[0,18-0,38]$ & & FOSpsf \\
\hline \multirow{3}{*}{$\begin{array}{l}\text { Carcasse } \\
\text { Jeunes Bovins }\end{array}$} & PCARCjb & & $x$ & $x$ & $x$ & & $x$ & $x$ & $x$ & $x$ & $\mathrm{H}$ & & $0,24[0,20-0,32]$ & & \\
\hline & CONFCjb & & $x$ & $x$ & $x$ & & $x$ & $x$ & $x$ & $x$ & $\mathrm{H}$ & & $0,21[0,16-0,24]$ & & CONFjbf \\
\hline & AGABAjb & & $\mathrm{x}$ & $x$ & $\mathrm{x}$ & & $x$ & $\mathrm{x}$ & $x$ & $\mathrm{x}$ & $\mathrm{H}$ & & $0,23[0,12-0,31]$ & & \\
\hline \multirow{4}{*}{$\begin{array}{l}\text { Carcasse } \\
\text { Veaux de } \\
\text { Boucherie }\end{array}$} & PCARCvb & & & $x$ & $x$ & & $x$ & & & & 1 & & $0,21[0,18-0,27]$ & & \\
\hline & CONFCvb & & & $x$ & $x$ & & $x$ & & & & 1 & & $0,12[0,05-0,27]$ & & CONFvbf \\
\hline & AGABAvb & & & $x$ & $x$ & & $x$ & & & & 1 & $\mathrm{x}$ & $0,27[0,22-0,37]$ & & \\
\hline & COUL & & & $\mathrm{x}$ & $x$ & & $x$ & & & & 1 & & $0,09[0,09-0,10]$ & & COULvbf \\
\hline
\end{tabular}

* les lettres identiques indiquent les caractères qui sont pris en compte dans un même modèle multi-caractère.

${ }^{* *}$ Envp = Effet d'environnement permanent pris en compte dans le modèle.

Abréviations : PN = Poids de Naissance ; CN = Condition de Naissance ; P120 = Poids-Age Type (PAT) à 120 jours ; P210 = PAT à 210 jours ; DM = Développement Musculaire au sevrage ; $\mathrm{DS}=$ Développement Squelettique au sevrage ; AF= Aptitudes Fonctionnelles ; $\mathrm{GC}=$ Grosseur des Canons $; \mathrm{REAC}=$ nombre de mouvements en bascule lors de la pesée sevrage $;$ COMP $=$ note de comportement

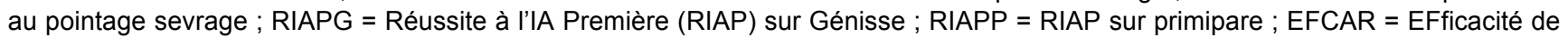
CARrière (nombre de veaux à 6,5 ans) ; $\mathrm{P} 12 \mathrm{M}=\mathrm{PAT}$ à 12 mois ; $\mathrm{P} 18 \mathrm{M}=\mathrm{PAT}$ à 18 mois ; $\mathrm{P} 24 \mathrm{M}=\mathrm{PAT}$ à 24 mois ; DM30M = Développement Musculaire à 30 mois ; $\mathrm{DS} 30 \mathrm{M}=$ Développement Squelettique à 30 mois ; $\mathrm{AF} 30 \mathrm{M}=\mathrm{AF}$ à 30 mois ; GC30M = GC à 30 mois ; PCARCjb = Poids de CARCasse (PCARC) Jeunes Bovins (JB); CONFCjb = CONFormation Carcasses (CONFC) JB ; AGABAjb = AGe à l'ABAttage (AGABA) JB ; PCARCvb = PCARC Veaux de Boucherie (VB); CONFCvb = CONFC VB ; AGABAvb = AGABA VB ; COUL = COULeur de viande. 
Par ailleurs, le recours à des modèles d'évaluation multi-caractères est nécessaire dans le cas d'une sélection par étapes successives au cours de la vie des candidats, afin d'éviter d'obtenir des estimations biaisées des valeurs génétiques. Par exemple, lorsqu'un premier tri des candidats est réalisé sur le caractère croissance avant sevrage, seuls les meilleurs animaux sont ensuite mis à la reproduction et produisent des veaux qui seront phénotypés pour les caractères de carcasse. Ignorer la première sélection lors de l'évaluation pour les caractères de carcasse conduirait à une estimation biaisée des valeurs génétiques sur ces caractères de carcasse. Seule une évaluation multicaractères permet d'éviter ce biais.

\section{d) Limites des évaluations polygéniques}

Bien que les programmes de sélection fondés sur l'utilisation de valeurs génétiques estimées à l'aide d'un modèle polygénique soient efficaces (comme en témoignent les progrès génétiques réalisés dans les populations sélectionnées ces dernières années), les évaluations génétiques polygéniques présentent des points faibles. Sous ce modèle, les performances des apparentés d'un jeune individu sans performance individuelle et sans descendant contrôlé permettent uniquement d'estimer les composantes parentales de sa valeur génétique additive, limitant la précision de la prédiction. Par ailleurs, un reproducteur transmet aléatoirement pour chaque QTL l'un ou l'autre de ses allèles à ses descendants. Des veaux issus d'un même taureau ou d'une même vache portent donc une fraction variable d'allèles identiques aux QTL transmis par ce parent. De fait, les coefficients de parenté calculés à partir des généalogies utilisés pour déterminer les corrélations entre valeurs génétiques des individus évalués ne peuvent pas refléter la proportion réelle d'allèles communs aux QTL entre apparentés, ce qui limite la qualité des estimations. Enfin, des QTL à effet moyen à fort ont été mis en évidence dans l'ensemble des espèces animales d'élevage, qui montrent que le déterminisme génétique sous-jacent au modèle polygénique infinitésimal peut parfois s'éloigner du déterminisme réel. La mise en œuvre d'évaluations génomiques permet de dépasser, au moins partiellement, ces limitations.

\section{2 / Évaluations génomiques}

\section{a) Principe des évaluations génomiques}

Un marqueur génétique est une séquence d'ADN « polymorphe », c'est-à-dire existant sous plusieurs formes (appelées «allèles »). Les marqueurs peuvent se situer dans des régions codantes ou non codantes de l'ADN, et ils n'ont en général pas d'effet biologique direct sur les phénotypes. Il y a plusieurs types de marqueurs génétiques, parmi lesquels les marqueurs SNP (« Single Nucleotide Polymorphism ») qui correspondent à une variation d'une seule paire de base de la séquence locale d'ADN, et qui existent en général sous la forme de 2 allèles. On trouve plusieurs millions de marqueurs SNP dans l'ADN des bovins, répartis sur l'ensemble du génome.

Meuwissen et al (2001) ont montré que i) en subdivisant le génome en un très grand nombre de segments chromosomiques dont la transmission d'une génération à l'autre peut être suivie grâce à un ou plusieurs marqueurs génétiques, puis $i$ ) en estimant l'effet de chaque segment à partir d'une population dite " de référence » constituée d'animaux à la fois phénotypés et génotypés pour ces marqueurs, et enfin iii) en sommant les effets des segments portés par n'importe quel nouvel animal génotypé, on obtient une estimation de la valeur génétique de cet animal. Le postulat de cette évaluation, appelée évaluation génomique, est que l'effet des allèles aux QTL (dont la localisation est en général inconnue) est « capté » par les marqueurs qui leur sont proches. Si les marqueurs sont en nombre suffisant et répartis sur l'ensemble du génome, et si la population de référence est de taille suffisamment grande, l'estimation de la valeur génétique d'un animal génotypé quelconque après une évaluation génomique, en particulier, un jeune individu ne disposant pas de performance propre ni de descendant, peut-être plus précise que son index polygénique sur ascendance (qui est la moyenne des index polygéniques de ses parents). La forte diminution du coût de génotypage des individus a permis d'envisager la mise en œuvre de telles procédures d'évaluations génomiques en bovins allaitants, comme cela a été le cas dans les populations bovines laitières.

Au cours des dernières années, plusieurs milliers d'individus ont été génotypés dans l'ensemble des populations bovines allaitantes françaises par les entreprises et les Organismes de Sélection, ou dans le cadre de projets de recherche ( Gembal », "Qualvigène »). La mise en commun de ces génotypes associés aux performances des individus a permis de mettre en place en 2015 des évaluations génomiques dans les populations Charolaise, Limousine et Blonde d'Aquitaine, pour les caractères mesurés en ferme à la naissance et au sevrage, ainsi que pour les caractères de croissance et de conformation de carcasse des jeunes bovins.

b) Modèle d'évaluation génomique actuellement mis en place dans les populations allaitantes françaises

L'évaluation génomique mise en place dans les populations allaitantes françaises repose sur une méthodologie en plusieurs étapes, réalisées intra-race.

La première étape consiste à estimer l'effet de chacun des 43801 marqueurs génétiques de la puce BovineSNP50 sur les performances des caractères à indexer, en s'appuyant sur une Population de Référence (PR) constituée de l'ensemble des individus génotypés avec phénotype pour les caractères évalués. Ce phénotype est obtenu en dé-régressant l'index polygénique de chaque individu de la PR selon la méthode proposée par Garrick et al (2009). Cet index dé-régressé est en quelque sorte un phénotype "synthétique » qui résume tous les phénotypes corrigés pour les effets de milieu de l'animal et de ses descendants, et qui sera d'autant plus précis que l'animal a de nombreux descendants phénotypés (par construction, au cours d'une évaluation polygénique utilisant les index dé-régressés comme phénotypes, on obtient les index polygéniques initiaux). Les effets des marqueurs sur chaque caractère sont ensuite estimés à partir des génotypes et des index dé-régressés de la PR, par une méthodologie statistique adaptée. Celle qui a été retenue est la méthode dite « Bayes-C » (Habier et al 2011), en considérant qu'une proportion de $2 \%$ des marqueurs ont un effet non nul sur les phénotypes et expliquent la totalité de la variabilité génétique du caractère (Tribout et al 2014). Ces effets estimés sont ensuite utilisés pour prédire la valeur génomique directe (DGV) de chaque animal génotypé de la population (candidat à la sélection ou reproducteur), en sommant simplement les effets estimés des allèles qu'il porte aux marqueurs.

À ce jour, une partie seulement des reproducteurs des populations bovines allaitantes est génotypée. La PR n'intègre donc qu'une partie de l'ensemble des données phénotypiques utilisées dans l'évaluation polygénique. Il s'agit alors dans une dernière étape de tirer profit des informations phénotypiques des apparentés non génotypés utilisés dans l'évaluation polygénique, mais non incluses dans la PR pour produire les index génomiques les plus précis possibles. La DGV de chaque individu est donc combinée à son index polygénique pour produire l'index génomique final aussi appelé GEBV («Genomic Enhanced Breeding Value »). Cette combinaison est réalisée selon une méthodologie permettant de tenir compte de la redondance partielle d'information entre les deux composantes (VanRaden et al 2009). Les pondérations respectives des composantes de la GEBV sont propres à chaque individu génotypé, et dépendent du CD de son index polygénique et de l'information supplémentaire apportée par les génotypes (Tribout et al 2015). Comme pour les index polygéniques, la 
précision d'une GEBV est appréciée au travers d'un CD variant entre 0 et 1 , calculé en cumulant les contributions des différentes sources d'information polygéniques et génomiques.

Les index génomiques produits par les évaluations génomiques sont plus précis que les index polygéniques. Le gain de CD est plus élevé pour les jeunes individus disposant uniquement d'information sur ascendance que pour les reproducteurs déjà bien connus sur descendance, pour lesquels l'information supplémentaire apportée par les génotypes n'est que marginale.

\section{3 / « Interbeef », évaluation génétique internationale des bovins allaitants}

La France a joué un rôle central depuis 2004 dans la mise en place de l'évaluation génétique internationale des bovins allaitants dite «Interbeef ». Cette évaluation est le résultat de la collaboration entre différents pays (France, Irlande, Royaume-Uni, Danemark, Suède, Norvège, Allemagne, Suisse, République Tchèque) qui fournissent les performances brutes et les généalogies de leurs animaux au Centre «Interbull » (localisé en Suède, sous-comité d'ICAR, «International Committee for Animal Recording », http://www.icar.org/). «Interbull 》 constitue ainsi une généalogie consolidée et réalise une évaluation génétique multicaractères (considérant le phénotype d'un individu dans différents pays comme autant de caractères différents) prenant en compte les spécificités des environnements de chaque pays et les interactions génotype-environnement. Sont diffusés ensuite, à chaque pays participant, les valeurs génétiques estimées (et leur précision) de tous les animaux de la généalogie commune, exprimées dans les échelles de chacun des pays (Venot et al 2014).

«Interbeef » ne porte actuellement que sur le poids au sevrage dans les races Charolaise et Limousine (première diffusion officielle début 2015). Il est prévu qu'elle soit étendue à l'avenir aux données de naissance, d'abattage et de fertilité, ainsi qu'à d'autres pays et d'autres races.

\section{4 / Mise en forme et expression des valeurs génétiques}

\section{a) Connexion entre élevages et compara- bilité des index}

Une connexion génétique est assurée par la présence dans plusieurs élevages de descendants de taureaux communs (connexion entre élevages) et par l'utilisation de mêmes reproducteurs au cours de plusieurs campagnes de naissance (connexion entre années). Sans connexion, on ne peut pas comparer objectivement les niveaux génétiques d'individus d'âges ou d'élevages différents. À la différence des races bovines laitières, le taux moyen d'insémination animale (IA) dans les populations allaitantes est faible (inférieur à 30\%). De ce fait, il peut exister des ensembles d'élevages mal « connectés » entre eux à cause de l'utilisation de taureaux d'IA pas ou peu apparentés ou par absence d'IA. Il est donc nécessaire d'être vigilant sur ce degré de connexion lors de la diffusion des index.

Dans l'évaluation génétique polygénique IBOVAL, le degré de connexion entre troupeaux et campagnes est apprécié au travers du CACO (Critère d'Admission au rang des troupeaux COnnectés) (Institut de l'Élevage et INRA, 2002 et 2005), calculé en s'appuyant sur les relations de parenté entre individus contrôlés sur les différents élevages au cours des 5 dernières campagnes. Le CACO, complété par un comptage du nombre de produits nés sur les élevages issus de taureaux connecteurs, permet d'établir la liste des élevages suffisamment connectés entre eux pour garantir une comparabilité des index des reproducteurs et jeunes individus entre eux. Pour les animaux présents dans les élevages dont la connexion est jugée insuffisante, seul un classement intra-élevage est fourni à l'éleveur.

\section{b) Standardisation des valeurs génétiques}

Dans la situation d'élevages suffisamment connectés au reste de la population, les index polygéniques et génomiques pour les caractères élémentaires (tableau 2) des individus présents sur un élevage connecté sont mis en forme de telle sorte que la valeur d'index 100 corresponde à l'index génétique moyen de la base de référence raciale et que 10 points d'index équivalent à 1 'écart type génétique du caractère considéré. La base de référence raciale est constituée des individus de race pure nés au cours des 5 à 12 dernières campagnes (selon le groupe de caractères évalués) disposant a minima de phénotypes pour tous les caractères mesurés au sevrage, ainsi que pour le caractère considéré dans les évaluations génétiques autres que « sevrage ». La base de référence raciale est réactualisée à chaque évaluation génétique. Les index génomiques et les index polygéniques sont exprimés dans la même échelle et sont directement comparables entre eux.

Pour un caractère donné, seuls les individus génotypés disposant d'un index polygénique individuel, ou à défaut sur ascendance, se voient calculer un index génomique GEBV accompagné d'un CD. Les individus génotypés sans index polygénique disposent quant à eux uniquement d'indicateurs génomiques, qui sont les valeurs génomiques directes (DGV) exprimées sous forme symbolique en 5 classes (dont les seuils correspondent aux GEBV minimum et maximum des 5 groupes de $20 \%$ des individus de la PR ordonnés selon leur GEBV) et non accompagnées de CD.

Le tableau 2 synthétise pour chaque caractère faisant l'objet d'une évaluation génétique, les races indexées, les paramètres génétiques, les modèles utilisés et les index publiés. Il donne donc la liste des principaux phénotypes collectés en ferme et valorisés.

\section{3 / Traduction des objectifs de sélection en index de synthèse, publication officielle des valeurs génétiques calculées et gestion des particularités génétiques}

Après avoir produit un certain nombre d'index élémentaires pour les différents critères à sélectionner, se pose la question de connaitre l'importance relative de ces critères.

\section{1 / Présentation de la méthodo- logie de calcul des objectifs de sélection et de l'outil OSIRIS}

La définition des objectifs de sélection des races est du ressort des Organismes de Sélection des races. Pour les aider à faire ce choix et afin de garantir une sélection des animaux économiquement supérieurs, des études économiques dans les systèmes de production de chaque race sont conduites par l'Institut de l'Élevage afin de hiérarchiser économiquement les caractères.

La méthode se décompose en deux phases :

i) Tout d'abord, il est nécessaire de décrire les systèmes d'élevages des animaux de la race étudiée ainsi que les performances de ces animaux, les prix des produits animaux et les coûts des aliments. Cette description s'appuie sur la description des cas-types des réseaux d'élevage principalement, mais aussi sur des études bibliographiques, des résultats de contrôles de performances, des résultats de stations expérimentales, des données provenant d'organisations de producteurs etc. À cette étape, il est important de veiller à la cohérence des données issues de sources nombreuses. 
Celles-ci permettent de calculer l'intérêt économique de chaque caractère grâce à des outils informatiques permettant de lier le phénotype des animaux dans un système avec les coûts et les recettes qui en découlent. Le projet OSIRIS (Guerrier 2013) soutenu par le Ministère en charge de 1'Agriculture et France Génétique Élevage a permis d'organiser et de développer les programmes nécessaires à ces études économiques. Désormais, les membres de l'équipe "objectifs de sélection » des deux unités mixtes technologiques de Jouy-en-Josas, anciennement appelée « génétique, génomique et gestion des populations » et nouvellement labellisée sous le nom d'e-BIS, et de
Toulouse, appelée "génétique et génomique des petits ruminants », utilisent des outils informatiques communs développés sous forme de modules, facilement adaptables et paramétrables selon les systèmes d'élevages étudiés. Les résultats produits par ces études économiques sont les poids économiques des caractères, exprimés en euros par unité du caractère.

ii) La seconde phase est la conversion des poids économiques des caractères et des orientations raciales en pondérations des index élémentaires dans des index dits « de synthèse », tout en prenant en compte les paramètres génétiques de ces caractères.

\section{2 / Présentation des index de synthèse}

Les index de synthèse sont des combinaisons linéaires des index élémentaires, issus d'études économiques synthétisant l'orientation définie par l'Organisme de Sélection de la race.

Il faut distinguer deux grandes familles d'index de synthèse : ceux issus d'évaluations portant sur des phénotypes recueillis en station et ceux issus d'évaluations portant sur des phénotypes recueillis en ferme. Le tableau 3 représente les différents index de synthèse pour les deux types d'outils, classés par

Tableau 3. Inventaire des index de synthèse disponibles.

\begin{tabular}{|c|c|c|c|}
\hline Index de synthèse & Composition & Nom en ferme & Nom en station \\
\hline Facilité de naissance & $\begin{array}{l}\text { Effet direct du poids et } \\
\text { conditions de naissance }\end{array}$ & IFNAIS & FNtest \\
\hline $\begin{array}{l}\text { Incidence de la mère } \\
\text { sur le poids au sevrage } \\
\text { du veau }\end{array}$ & $\begin{array}{l}\text { Effet maternel et moitié } \\
\text { de l'effet direct du poids } \\
\text { sevrage du veau }\end{array}$ & MERPsev & I \\
\hline $\begin{array}{l}\text { Croissance et } \\
\text { morphologie }\end{array}$ & $\begin{array}{l}\text { Facilité de naissance, } \\
\text { croissance, morphologie } \\
\text { en effet direct }\end{array}$ & ISEVR au sevrage & $\begin{array}{l}\text { IMOCR en post-sevrage } \\
\text { pour les stations de } \\
\text { contrôle sur } \\
\text { descendance des } \\
\text { qualités maternelles, les } \\
\text { stations de contrôle } \\
\text { individuel et les stations } \\
\text { d'évaluation }\end{array}$ \\
\hline $\begin{array}{l}\text { Aptitudes bouchères } \\
\text { en vif }\end{array}$ & $\begin{array}{l}\text { Croissance et } \\
\text { développement } \\
\text { musculaire } \\
\end{array}$ & I & $\begin{array}{l}\text { IABV pour les stations de } \\
\text { contrôle individuel et les } \\
\text { stations d'évaluation }\end{array}$ \\
\hline $\begin{array}{l}\text { Croissance de la } \\
\text { carcasse }\end{array}$ & $\begin{array}{l}\text { Poids de carcasse et âge } \\
\text { à l'abattage }\end{array}$ & $\begin{array}{l}\text { ICRCjbf pour les jeunes } \\
\text { bovins } \\
\text { et ICRCvbf pour les } \\
\text { veaux de boucherie }\end{array}$ & 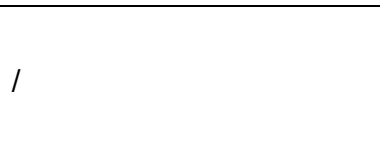 \\
\hline $\begin{array}{l}\text { Aptitudes bouchères } \\
\text { en carcasse }\end{array}$ & $\begin{array}{l}\text { Croissance, conformation } \\
\text { et couleur des carcasses }\end{array}$ & $\begin{array}{l}\text { IABjbf pour les jeunes } \\
\text { bovins } \\
\text { et IABvbf pour les veaux } \\
\text { de boucherie }\end{array}$ & I \\
\hline Fertilité & $\begin{array}{l}\text { Précocité, viabilité et } \\
\text { réussite à l'IA }\end{array}$ & 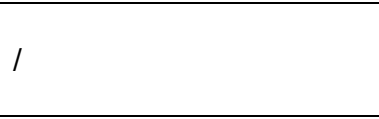 & $\begin{array}{l}\text { IFER en contrôle sur } \\
\text { descendance des } \\
\text { qualités maternelles }\end{array}$ \\
\hline Vêlage & $\begin{array}{l}\text { Préparation au vêlage, } \\
\text { ouverture pelvienne, } \\
\text { poids après vêlage et } \\
\text { incidence de la mère sur } \\
\text { le poids naissance du } \\
\text { veau }\end{array}$ & I & $\begin{array}{l}\text { IVEL en contrôle sur } \\
\text { descendance des } \\
\text { qualités maternelles }\end{array}$ \\
\hline $\begin{array}{l}\text { Croissance et } \\
\text { morphologie du produit }\end{array}$ & $\begin{array}{l}\text { Production laitière, } \\
\text { incidence de la mère sur } \\
\text { le P120, P180 et le DM } \\
\text { du veau }\end{array}$ & I & $\begin{array}{l}\text { IMER en contrôle sur } \\
\text { descendance des } \\
\text { qualités maternelles }\end{array}$ \\
\hline Qualités maternelles & $\begin{array}{l}\text { facilité de naissance, } \\
\text { croissance, morphologie } \\
\text { en effet direct et maternel }\end{array}$ & IVMAT & $\begin{array}{l}\text { IQM en contrôle sur } \\
\text { descendance des } \\
\text { qualités maternelles }\end{array}$ \\
\hline Synthèse raciale & Variable selon les races & ISU & I \\
\hline
\end{tabular}

$\mathrm{P} 120$ = poids-âge type à 120 jours, $\mathrm{P} 180$ = poids-âge type à 180 jours et DM = Développement Musculaire avant le sevrage. $/$ = index de synthèse non disponible. 
catégorie d'aptitudes. Leur standardisation est effectuée de manière à ce que leur écart-type, mesuré sur les animaux des bases de référence, soit de 8 points. L'index de synthèse IVMAT, pour « index de valeur maternelle synthétique au sevrage ", est la synthèse calculée en ferme, censée orienter les races pour la production au sevrage. Il est composé des index élémentaires au sevrage traduisant les effets directs et maternels.

Tous les index dits «station» (car obtenus à partir des phénotypes collectés en station) ne sont pas disponibles pour toutes les races. Par exemple, les évaluations des qualités maternelles par contrôle de la descendance n'existent qu'en races Limousine et Blonde d'Aquitaine. En ferme également, toutes les races ne disposent pas de toutes les évaluations génétiques IBOVAL, comme l'illustre le tableau 4. Seules 3 races (Charolaise, Limousine et Rouge des Prés) disposent de toutes ces évaluations.

\section{3 / Les pondérations des index de synthèse}

Les pondérations des index de synthèse varient selon la race et selon leur outil de sélection individuelle des mâles en station (SE et stations de CI), même si les tendances sont les mêmes. Les conséquences de la sélection sur ces index de synthèse sont appelées "réponses à la sélection ». Ces réponses sont la différence des moyennes d'index entre tous les animaux qui étaient candidats et ceux qui ont été retenus d'après la valeur de leur index de synthèse et d'après l'intensité de sélection souhaité.

À titre d'illustration, la figure 2 présente les réponses à la sélection de l'index de synthèse « morphologie-croissance » (IMOCR), en station de CI pour différentes races, actualisés en 2011 et 2015. Cet index de synthèse intègre les index élémentaires obtenus en station de CI : CRci (croissance), DMci (développement musculaire), DSci (développement squelettique), EFAci (efficacité alimentaire), AFci (aptitudes fonctionnelles), QRci (qualités de race), IFNAIS_asc (facilité de naissance en ferme calculée sur ascendance) et OPci (ouverture pelvienne). On notera qu'en races Charolaise et Rouge des Prés, il n'y a pas de calcul d'index sur les qualités de race. On constate que les animaux sélectionnés à la suite d'un CI sont des animaux avec une forte croissance, un fort développe- ment musculaire et du développement squelettique (et de l'efficacité alimentaire en Rouge des Prés). Les animaux sélectionnés n'améliorent que légèrement les autres critères, voire détériorent légèrement la facilité de naissance.

\section{4 / La publication officielle des index issus de l'évaluation génétique en ferme IBOVAL}

Les normes générales relatives à la diffusion des index standardisés (cf. § $2.4 b$ ) sont décidées en accord avec tous les partenaires au sein de France Génétique Élevage.

Les index polygéniques des femelles ne sont généralement jamais connus avec précision compte tenu du faible nombre de descendants qu'elles sont susceptibles d'engendrer. Néanmoins, pour fournir des éléments de tri relativement pertinents sur des lots de femelles, il est utile de disposer de tout ou partie des index de ces dernières. Pour une femelle, l'index élémentaire issu de l'estimation des effets directs (ex. " index croissance sevrage ", CRsev) est publié dès lors que son ascendance est connue et qu'elle dispose d'une performance propre (pour

Tableau 4. Effectifs de femelles et de taureaux publiés pour IBOVAL 2016_02 (hiver 2016) par index ou type d'évaluation génétique.

\begin{tabular}{|c|c|c|c|c|c|c|c|c|c|c|c|c|c|c|c|}
\hline \multirow{3}{*}{ Races } & \multicolumn{4}{|c|}{ Femelles avec index publiés } & \multicolumn{11}{|c|}{ Taureaux avec index publiés par type d'évaluation génétique } \\
\hline & \multirow{2}{*}{$\begin{array}{l}\text { Jusqu'au } \\
\text { sevrage }\end{array}$} & \multicolumn{3}{|c|}{ Post-sevrage } & \multirow{2}{*}{$\begin{array}{l}A B \\
\text { vbf }\end{array}$} & \multicolumn{5}{|c|}{ Jusqu'au sevrage * } & \multicolumn{5}{|c|}{ Post-sevrage } \\
\hline & & CRpsf & Mpsf & IABjbf & & REAC & COMP & ISEVR & AVel & IVMAT & CRpsf & f Mpsf & RIAP & EFCAR & ABjbf* \\
\hline AUB & 260041 & I & l & l & I & 24 & 54 & 1981 & 1185 & 890 & l & I & 30 & 860 & I \\
\hline BAZ & 9513 & 1925 & I & I & I & 3 & 1 & 41 & 30 & 26 & 23 & I & I & 26 & I \\
\hline BLA & 883194 & 83963 & 1 & 47947 & 78 & 132 & 191 & 4995 & 3339 & 2717 & 827 & 1 & 150 & 2256 & 929 \\
\hline $\mathrm{CHA}$ & 2753328 & 351885 & 475524 & 263643 & 280 & 205 & 495 & 25607 & 13816 & 11808 & 3191 & 4055 & 625 & 7173 & 4026 \\
\hline GAS & 52257 & I & I & I & I & 0 & 4 & 284 & 194 & 145 & I & I & I & 118 & I \\
\hline INRA & 1 & I & I & I & 78 & 1 & I & I & I & I & I & 1 & 1 & I & I \\
\hline LIM & 1585350 & 96595 & 368334 & 111373 & 258 & 593 & 1029 & 17858 & 9941 & 8159 & 1414 & 5051 & 203 & 5436 & 2835 \\
\hline PAR & 134363 & 15271 & 1 & 25731 & 1 & 152 & 176 & 1644 & 1148 & 986 & 253 & I & 52 & 704 & 642 \\
\hline ROU & 132827 & 11341 & 15862 & 18700 & 1 & 16 & 64 & 1039 & 733 & 622 & 157 & 170 & 47 & 469 & 364 \\
\hline SAL & 261460 & I & I & 15736 & I & 76 & 140 & 2569 & 1474 & 1265 & 1 & I & 26 & 840 & 266 \\
\hline Total & 6072333 & 560980 & 859720 & 483130 & 666 & 1201 & 2154 & 5018 & 31860 & 26618 & 5865 & 9276 & 1113 & 17882 & 9062 \\
\hline
\end{tabular}

AUB = AUBrac, BAZ = BAZadaise, CHA = CHArolaise, GAS = GASconne, INRA = INRA 95, LIM = LIMousine, PAR = PARthenaise, $\mathrm{ROU}=\mathrm{ROUge}$ des Prés, $\mathrm{SAL}=$ SALers .

Pour les femelles:

Jusqu'au sevrage : au moins 1 index pour les caractères de naissance - comportement - croissance - morphologie.

Post-sevrage : croissance (CRpsf), Mpsf = morphologie post-sevrage : 5 index par race, IABjbf=synthèse Aptitudes Bouchères sur la production de jeunes bovins en ferme.

Pour les taureaux : successivement ABvbf : croissance, conformation, couleur et synthèse pour les veaux de boucherie ; 'docilité' = réactivité des veaux au sevrage (REACsev) et comportement au pointage (COMPsev) ; ISEVR sous-entend publication également de tous les index élémentaires qui le composent (IFNAIS, CRsev, DMsev, DSsev, FOSsev) ; avec AVel en plus ; ou sur l'ensemble des index jusqu'au sevrage (IVMAT, y compris MERPsev, incidence de la mère au sevrage) ; ou bien sur l'une ou l'autre des 5 indexations post-sevrage : CRpsf, Mpsf, RIAPgef (réussite à l'IA première sur génisse), EFCAR (efficacité carrière) et ABjbf (croissance, conformation et synthèse).

* possibilité d'avoir des animaux publiés avec un index génomique (13 index génomiques possibles au total, hors REACsev et COMPsev).

/ : Pas d'indexation IBOVAL pour ce(s) caractère(s) dans cette race. 
Figure 2. Réponses à la sélection en point d'index de l'index de synthèse IMOCR (" morphologie et croissance ") en station de contrôle individuel pour les races Blonde d'Aquitaine, Rouge des Prés, Charolaise et Limousine.

Avec : $\mathrm{Cl}$ : Contrôle Individuel.

1 écart-type de chaque index $=10$ points.

Réponse à la sélection : différence des moyennes d'index entre tous les animaux qui étaient candidats et ceux qui ont été retenus d'après la valeur de leur index de synthèse et d'après l'intensité de sélection souhaité.

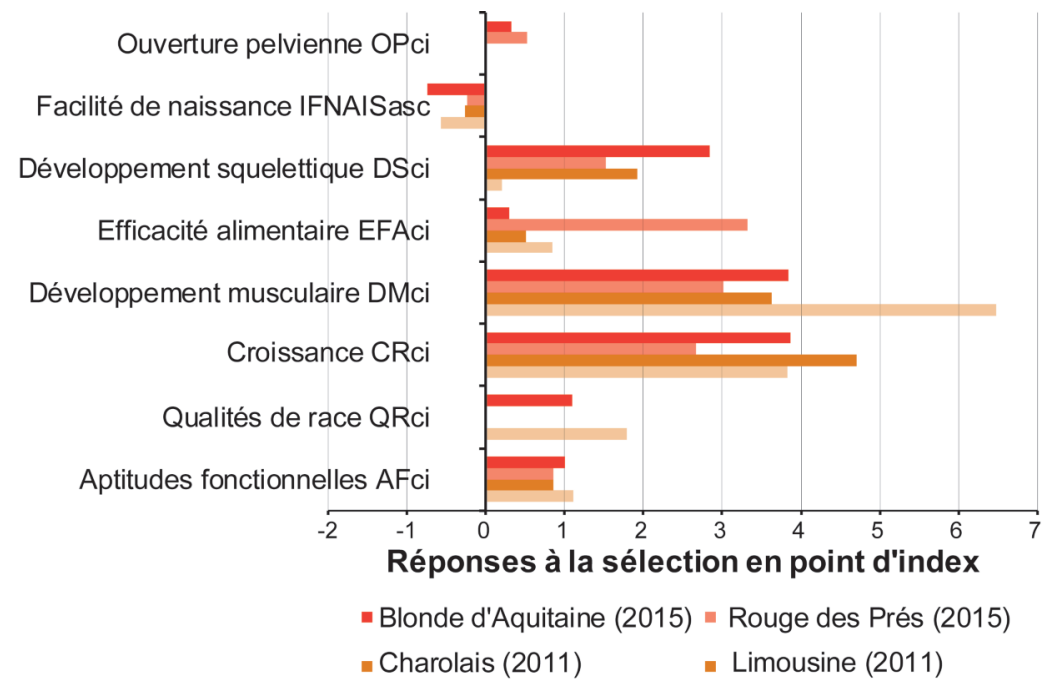

l'exemple P210). Pour cette même femelle, l'index élémentaire issu de l'estimation des effets maternels (ex. « index aptitude laitière », ALait) est publié si et seulement si l'index issu de l'estimation des effets directs est luiaussi publié (ex. « index croissance sevrage », CRsev). Ces choix aboutissent à un nombre conséquent de femelles avec index publiés (tableau 4). Par ailleurs, en fonction de la qualité de la connexion (cf. § $2.4 a$ ), l'expression des index d'un troupeau s'effectue soit de manière commune à l'ensemble de la race (troupeau considéré suffisamment connecté), soit à l'échelle du troupeau seul. Une femelle peut être connue sur un maximum de 19 index IBOVAL, 12 de la naissance jusqu'au sevrage et 7 au-delà du sevrage.

Pour les index polygéniques des mâles, les choix effectués jusqu'à présent ont été d'apporter une garantie plus importante à l'utilisateur, compte tenu du plus grand nombre de produits que chacun est susceptible d'engendrer. Cette précaution se traduit par des exigences minimales à la fois en termes d'effectifs de produits (effets directs) et/ou de produits issus des filles (effets maternels) pris en compte dans l'évaluation et de CD minimal. Ces seuils au niveau des produits permettent de s'assurer que les informations sur descendance ont un certain poids dans le CD. Par ailleurs, les index des taureaux ne sont publiés que s'ils ont des produits et des filles présents dans des élevages « connectés » (cf. $§ 2.4 a$ ). Ainsi, le nombre de taureaux avec index publiés est plus restreint que pour les femelles (tableau 4), mais avec une précision moyenne plus élevée. Un taureau peut être connu sur un maximum de 27 index IBOVAL, 16 de la naissance jusqu'au sevrage et 11 au-delà du sevrage. Chaque année, toutes races confondues, les index de plus de 3000 nouveaux taureaux sont publiables.

Avec l'arrivée de la prise en compte d'informations génomiques dans l'estimation de la valeur génétiques des animaux, pour les mâles, la notion de CD est renforcée au détriment de l'exigence sur le nombre de produits pris en compte (suppression des seuils). Une publication beaucoup plus précoce des index génomiques GEBV des taureaux est permise par ces changements mis en place début 2017 dans la chaîne de publication des évaluations officielles. Pour les femelles, tous les index génomiques GEBV calculés sont diffusés.

\section{5 / Gestion des particularités génétiques à l'heure de la géno- mique}

Sous le terme de particularités génétiques, nous faisons référence à des mutations ayant pour conséquence l'observation de phénotypes particuliers (cf. Boichard et al 2016), voire parfois spectaculaires, tels que le phénotype culard. analysée que si des animaux affectés ou porteurs sont signalés à l'Observatoire National des Anomalies Bovines (https:// www.onab.fr/) afin de collecter un ensemble d'éléments tels que l'identité des animaux, les phénotypes aussi précis que possible et le matériel biologique Une particularité génétique ne peut être nécessaire au génotypage de ces animaux (Grohs et al 2016). Ces résultats sont d'importance car ils peuvent imposer la réforme d'un animal même si ses index sont favorables. Par exemple, dans une race dont l'Organisme de Sélection ne veut pas sélectionner d'animaux ayant des anomalies chromosomiques comme des translocations, aucun taureau porteur n'est autorisé à la mise à la reproduction, quel que soit son niveau d'index. Cependant les particularités génétiques ne sont pas forcément négatives : la mutation « sans corne » par exemple permet de sélectionner des animaux qui naturellement ne seront pas pourvus de cornes, ce qui évitera leur écornage.

Les informations sur ces particularités génétiques peuvent être obtenues par génotypage. Avec le développement du génotypage dans les principales races bovines, la gestion des particularités génétiques est simplifiée. Si la position de la mutation responsable du phénotype est connue précisément, un marqueur correspondant peut être proposé à la commercialisation via les puces à ADN. Dans ce cas, la détection de la mutation est quasi-certaine, voire certaine si c'est la mutation elle-même qui est utilisée. Dans le cas contraire, un test sur haplotype consistant en la recherche d'une suite plus ou moins longue de marqueurs non spécifiques de la mutation, mais partagés par les animaux porteurs, peut être mis en place de la même façon. Il est cependant moins fiable.

À l'heure actuelle, en bovins allaitants, entre un et cinq tests sont disponibles selon la race, y compris les races ne disposant pas d'évaluation génomique. Ce nombre devrait connaître une augmentation importante dans les prochaines années en raison du développement de la sélection génomique. Les résultats de ces tests pour les animaux reproducteurs sont autant d'informations à prendre en compte lors des plans d'accouplements mis en place à chaque campagne de reproduction. Une bonne prise en compte de ces résultats permet de minimiser le nombre d'animaux qui exprimeront les phénotypes pour les mutations délétères et de maximiser le nombre d'animaux qui exprimeront les phénotypes pour les mutations d'intérêt. En fonction des races et/ou des éleveurs, les choix des mutations d'intérêt ne sont pas forcément les mêmes, ce qui est le cas par exemple pour le phénotype culard. Des éleveurs vendeurs de reproducteurs voudront qu'aucune de leurs reproductrices n'en soit porteuse afin d'éviter des problèmes lors du vêlage, alors que des éleveurs engraisseurs peuvent vouloir des animaux porteurs afin d'avoir des carcasses mieux conformées.

Les mutations délétères nécessitent une gestion fine : une volonté d'éradication 
des animaux porteurs de mutations délétères risquerait d'entraîner une perte de variabilité génétique irrémédiable pouvant être pénalisante pour la race en termes de sélection. La mise en place de plans de gestions adaptés est préférable sur le long terme.

\section{4 / Impact des programmes de sélection}

\section{1 / Évolutions génétiques obser- vées dans la population}

Ces 15 dernières années, le poids à âge type 210 jours des mâles des races Limousine, Charolaise et Blonde d'Aquitaine a augmenté d'environ $30 \mathrm{~kg}$ en moyenne. Une partie de ce gain provient de l'amélioration génétique des animaux : à titre d'exemple, les évolutions au cours des 15 dernières années du niveau génétique estimé sur ce caractère (effet génétique direct - index croissance au sevrage CRsev) sont présentées sur la figure 3 .

Une évolution positive, d'amplitude variable, est observée dans toutes les races : entre 2000 et 2015 , le poids à 210 jours a augmenté de 3,5 kg (Aubrac,
Bazadaise) à $16 \mathrm{~kg}$ (Limousine), représentant un accroissement de $27 \%$ (Bazadaise) à $102 \%$ (Limousine) d'écart-type génétique, soit un progrès génétique annuel de $2 \%$ d'écart-type génétique en Bazadaise à $7 \%$ en Limousine. Ces variations entre races peuvent s'expliquer par des différences de pondération de l'index CRsev dans les objectifs de sélection de chaque race (pondération plus faible par exemple en race Aubrac qui cherche à maitriser l'augmentation du poids des vaches), par des différences de tailles de base de sélection (limitée par exemple dans le cas de la race Bazadaise) ou par les spécificités du schéma des races (pression forte par exemple en race Limousine sur la croissance à l'entrée des veaux dans la station d'évaluation de Lanaud, Haute-Vienne).

La sélection des animaux sur la croissance au sevrage a toutefois entraîné une augmentation conjointe défavorable du poids de naissance des veaux (entre 0,2 et $1 \mathrm{~kg}$ depuis 2000 , soit entre 7 et $34 \%$ d'écart-type génétique selon les races, sauf pour la race Rouge des Prés dont le poids naissance est resté stable depuis 2000) et une dégradation des conditions de naissance pour certaines races. Un ajustement des pondérations des com-

Figure 3. Progrès génétique estimé pour le poids-âge-type au sevrage depuis 2000 dans les 9 races évaluées (moyenne par année de naissance des valeurs génétiques directes CRsev pour les animaux avec performances) - référence IBOVAL 2017_01.

IBOVAL = indexation bovine allaitante en ferme.

CRsev = index croissance au sevrage.

Liste des races et de leur écart-type génétique arrondi à l'unité $\left(\mathrm{s}_{\mathrm{g}}\right)$ :

AUB = Aubrac $\left(\mathrm{s}_{\mathrm{g}}=9 \mathrm{~kg}\right), \mathrm{BAZ}=$ Bazadaise $\left(\mathrm{s}_{\mathrm{g}}=13 \mathrm{~kg}\right), \mathrm{BLA}=$ Blonde d'Aquitaine $\left(\mathrm{s}_{\mathrm{g}}=16 \mathrm{~kg}\right), \mathrm{CHA}=$ Charolaise $\left(\mathrm{s}_{\mathrm{g}}=16 \mathrm{~kg}\right), \mathrm{GAS}=$ Gasconne $\left(\mathrm{s}_{\mathrm{g}}=12 \mathrm{~kg}\right), \mathrm{LIM}=$ Limousine $\left(\mathrm{s}_{\mathrm{g}}=16 \mathrm{~kg}\right), \mathrm{PAR}=$ Parthenaise $\left(\mathrm{s}_{\mathrm{g}}=14 \mathrm{~kg}\right), \mathrm{ROU}=$ Rouge des Prés $\left(\mathrm{s}_{\mathrm{g}}=14 \mathrm{~kg}\right), \mathrm{SAL}=$ Salers $\left(\mathrm{s}_{\mathrm{g}}=12 \mathrm{~kg}\right)$.

Toutes les races sont mises artificiellement à $0 \mathrm{~kg}$ pour l'année 2000. Seule la pente est comparable entre les races.

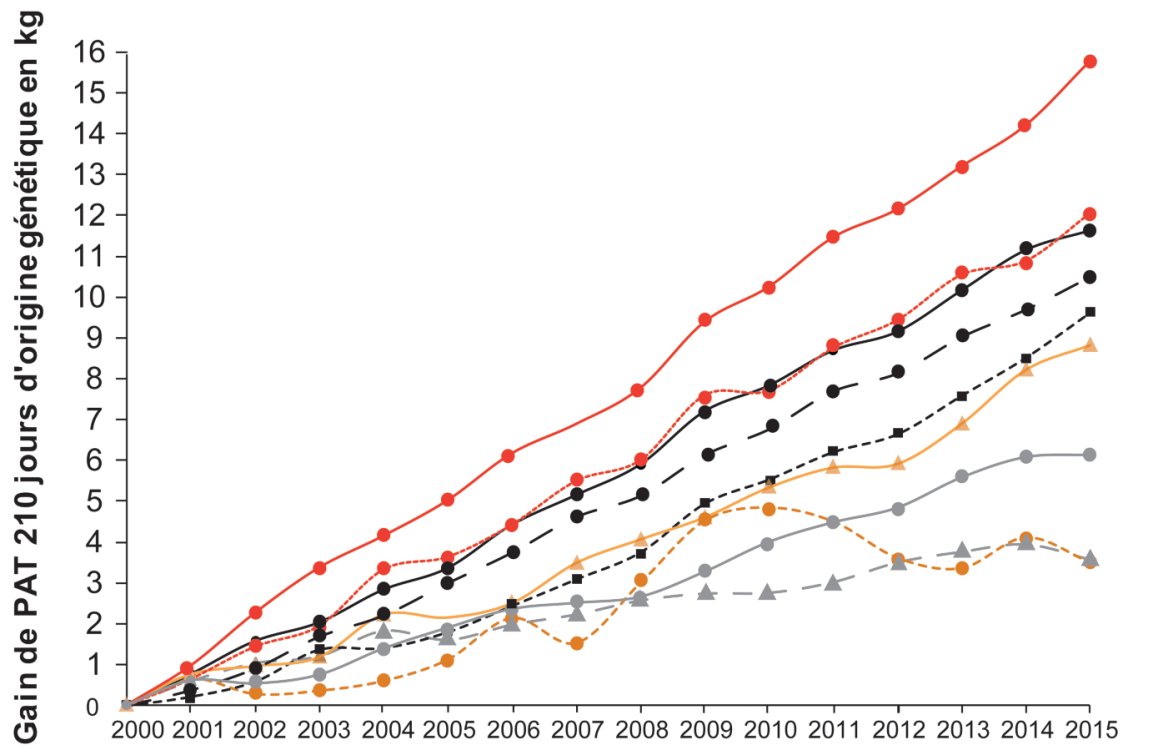

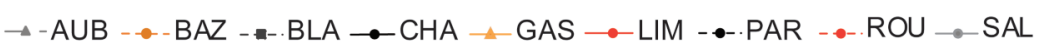

posantes des objectifs de sélection raciaux pourrait permettre à l'avenir de limiter ces évolutions génétiques défavorables.

\section{2 / Diffusion du progrès géné- tique dans la population}

L'objectif d'un programme de sélection est également de diffuser le progrès génétique généré dans l'ensemble de la population associée. L'insémination animale est un outil qui peut permettre une diffusion rapide et à grande échelle du progrès réalisé. Toutefois, l'utilisation de l'IA dans les populations allaitantes françaises reste limitée : en 2015 , seules $12 \%$ des naissances, toutes races confondues, sont issues de taureaux d'IA (figure 4). L'IA ne peut donc être le seul vecteur de diffusion du progrès génétique. La vente de taureaux de monte naturelle (MN) permet aussi cette diffusion : ils sont près de 200000 en activité et contribuent à $88 \%$ des naissances.

a) Analyse des flux de reproducteurs allaitants

Un suivi précis de la diffusion du progrès génétique dans les populations allaitantes est réalisé chaque année par l'Institut de l'Élevage. Pour la campagne 2015 (naissances du 01/08/14 au 31/07/15), 3815000 naissances ont pu être analysées : pour $88 \%$ de ces naissances, les éleveurs ont indiqué le père des veaux (64\% sont simplement notifiés et $24 \%$ sont certifiés par la CPB). L'analyse de ces pères est donc pertinente vu ce taux de pères déclarés. Les résultats sont donnés ci-dessous pour les veaux nés durant cette campagne 2015 (Institut de l'Élevage 2017).

Les élevages en contrôle de performances utilisent les taureaux d'IA en proportion plus importante que les autres élevages : $24 \%$ de leurs naissances sont issues d'IA vs $6 \%$ pour les élevages hors base de sélection (cf. $\S 1$, voir définition de la base de sélection). Par conséquent, on peut identifier un échelon de diffusion indirecte des taureaux d'IA via leurs fils utilisés en monte naturelle, qui représentent $14 \%$ des naissances totales. Ainsi $26 \%$ des naissances sont issues directement ou indirectement des taureaux d'IA (17\% en race Limousine, $31 \%$ en Charolaise et $35 \%$ en Blonde d'Aquitaine). L'utilisation de l'IA est historiquement plus forte en race Blonde d'Aquitaine du fait entre autres de la taille plus réduite des exploitations. De même, cette utilisation de l'IA est plus forte en race Charolaise par rapport à la race Limousine.

La diffusion du progrès génétique en races allaitantes est aussi réalisée au travers des jeunes taureaux évalués en station, avec $6 \%$ des naissances allaitantes 
Figure 4. Typologie des pères utilisés toutes races confondues en pourcentage des naissances de veaux issus du troupeau bovin allaitant en 2015.

MN : Monte Naturelle.

VA4 : contrôle de performances avec pesées.

$V A O$ : contrôle de performances sans pesée.

CPB : Certification de la Parenté Bovine.

Hors base : élevages à l'identification pérenne généralisée, non adhérents à la CPB.

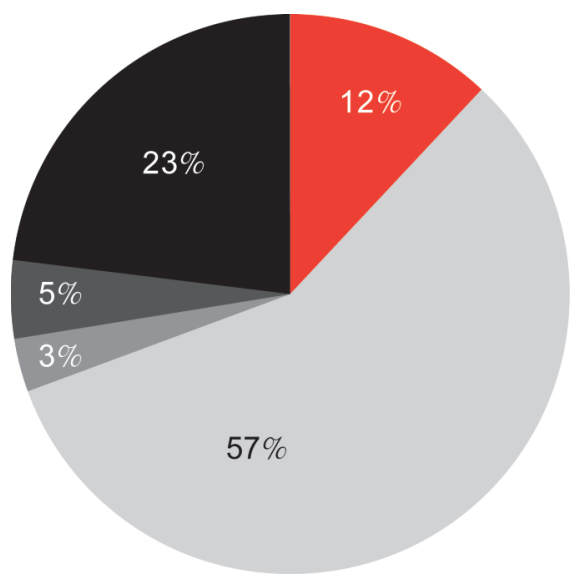

- Père d'insémination animale

$\square \mathrm{MN}$ : Père issu du VA4

- $\mathrm{MN}$ : Père issu du VAO

- $\mathrm{MN}$ : Père issu de CPB

- MN : Père issu d'élevage Hors base

en race pure directement issues de ces taureaux. L'impact direct des stations peut donc paraître faible, mais comme pour l'utilisation de l'IA, ces taureaux sont ensuite utilisés dans les élevages de la base de sélection qui revendent ultérieurement des descendants de ces taureaux aux autres élevages. Ainsi 16\% des naissances totales en race pure sont issues directement ou indirectement des taureaux contrôlés en station : cette proportion varie de $5 \%$ pour la race Charolaise à plus de $25 \%$ pour les races Limousine, Aubrac, Salers, Parthenaise, Rouge des Prés et Gasconne. Elle est faible en race Charolaise, car les effectifs contrôlés en SE et stations de CI (765 en 2015, tableau 1) sont faibles au regard de la demande en taureaux de MN. Cette proportion est plus conséquente notamment dans les races rustiques (Aubrac, Salers et Gasconne) dans lesquelles la demande en taureaux de MN est beaucoup moins importante.

En analysant l'origine des pères des veaux issus de $\mathrm{MN}$, on voit que par rapport à l'ensemble des naissances toutes races confondues (figure 4) :

i) $60 \%$ des pères proviennent d'élevages adhérents des organismes «Bovins Croissance » (57\% d'élevages en formule avec pesées et $3 \%$ d'élevages en formule sans pesée) ;

ii) seuls $5 \%$ des pères viennent d'élevages seulement adhérents de la $\mathrm{CPB}$.

En ajoutant les pères d'IA, $77 \%$ des veaux sont donc issus de pères en provenance de la base de sélection (cf. $\S 1$, strates d'éleveurs adhérents à la CPB et au contrôle de performances). On peut donc dire que la base de sélection diffuse efficacement le progrès génétique généré. Une amélioration supplémentaire de la diffusion pourrait encore être réalisée sur les $23 \%$ de veaux dont les pères sont issus d'élevages hors base de sélection.

Les proportions des différents types de reproducteurs utilisés varient selon les différentes strates d'élevages : la pyramide présentée en figure 5 indique la structure des populations allaitantes (contrôle de performances en formules VA4 et VA0, CPB et hors base de sélection). Les flèches de même couleur montrent leurs sources d'approvisionnement en reproducteurs. Par exemple, les naissances dans les élevages hors base de sélection sont issues pour $62 \%$ de reproducteurs provenant d'élevages du contrôle de performances avec pesées (VA4), pour $3 \%$ de reproducteurs provenant d'élevages au contrôle de performances sans pesée (VA0), etc.

Pour les élevages adhérents au contrôle de performances avec pesées (VA4), les reproducteurs utilisés proviennent presque exclusivement de la même strate $(96 \%$ des naissances).

On constate donc que les échanges de mâles se font préférentiellement à partir d'éleveurs très engagés dans la collecte de performances et disposant de références (index IBOVAL).

\section{b) Analyse qualitative des reproducteurs utilisés}

Les proportions des veaux de race pure (soit $84 \%$ de l'ensemble des naissances annuelles) issus de pères disposant d'un index IBOVAL varient selon les groupes de caractères considérés : $80 \%$ des veaux pour les caractères de facilité de naissance et d'aptitude au vêlage, 71\% des veaux pour l'ensemble des caractères directs et maternels évalués au sevrage, et $67 \%$ des veaux pour les caractères d'aptitudes bouchères sur production de jeunes bovins.

Le niveau génétique moyen de ces pères (toutes races confondues) pour la campagne 2015 est présenté sur la figure 6 (moyennes des index, pondérés par le nombre de veaux de chaque père).

Bien que les orientations de sélection de chacune des races diffèrent, on constate que les éleveurs ont globalement utilisé des taureaux améliorateurs (c'està-dire, dont l'index était supérieur à celui de la base raciale de référence) pour le développement musculaire et la conformation (sevrage, post-sevrage et jeunes bovins), ainsi que pour le développement squelettique au sevrage. En revanche, la valeur génétique pour la facilité de naissance, la finesse d'os, les aptitudes au vêlage et à l'allaitement, ou encore la réussite à l'IA ne semblent globalement pas être des critères pris en compte par les éleveurs lors du choix de leurs reproducteurs, puisque les taureaux utilisés se sont révélés moyens, voire plus mauvais que la base raciale de référence pour ces objectifs. Ceci peut sembler paradoxal compte tenu de l'orientation affichée de la filière allaitante d'améliorer à la fois le potentiel de croissance, les aptitudes bouchères et les aptitudes maternelles des populations, mais peut s'expliquer par une vision différente des besoins entre les Organismes de Sélection et les éleveurs utilisateurs.

\section{5 / Implications et perspectives}

Le dispositif d'amélioration génétique des populations bovines allaitantes françaises a beaucoup évolué au cours des dernières décennies, tant en matière d'outils mis en place que dans la valorisation des données produites. Entre 2007 et 2016, le nombre d'évaluations génétiques de groupes de caractères des 9 races allaitantes est passé de 9 à 53 pour les évaluations polygéniques et de 0 à 6 pour les évaluations génomiques. Toutes ces évolutions ont généré et génèreront un progrès génétique dont les éleveurs allaitants sont les bénéficiaires.

La composition et les pondérations des index de synthèse en ferme doivent être revues suite aux développements de nouvelles évaluations génétiques pour des caractères post-sevrage, et afin de contrer les évolutions génétiques défavorables observées sur le poids à la naissance et les conditions de naissance, préjudiciables à un élevage durable. Il faut également s'adapter aux évolutions du contexte économique, de la demande de la 
Figure 5. Illustration des flux de reproducteurs mâles entre les différentes strates d'éleveurs en 2015, toutes races confondues.

VA4 : contrôle de performances avec pesées.

VAO : contrôle de performances sans pesée.

CPB : Certification de la Parenté Bovine.

Hors base : élevages à l'identification pérenne généralisée, non adhérents à la CPB.

Les flux qui alimentent la même strate d'éleveurs sont représentés avec la même couleur.

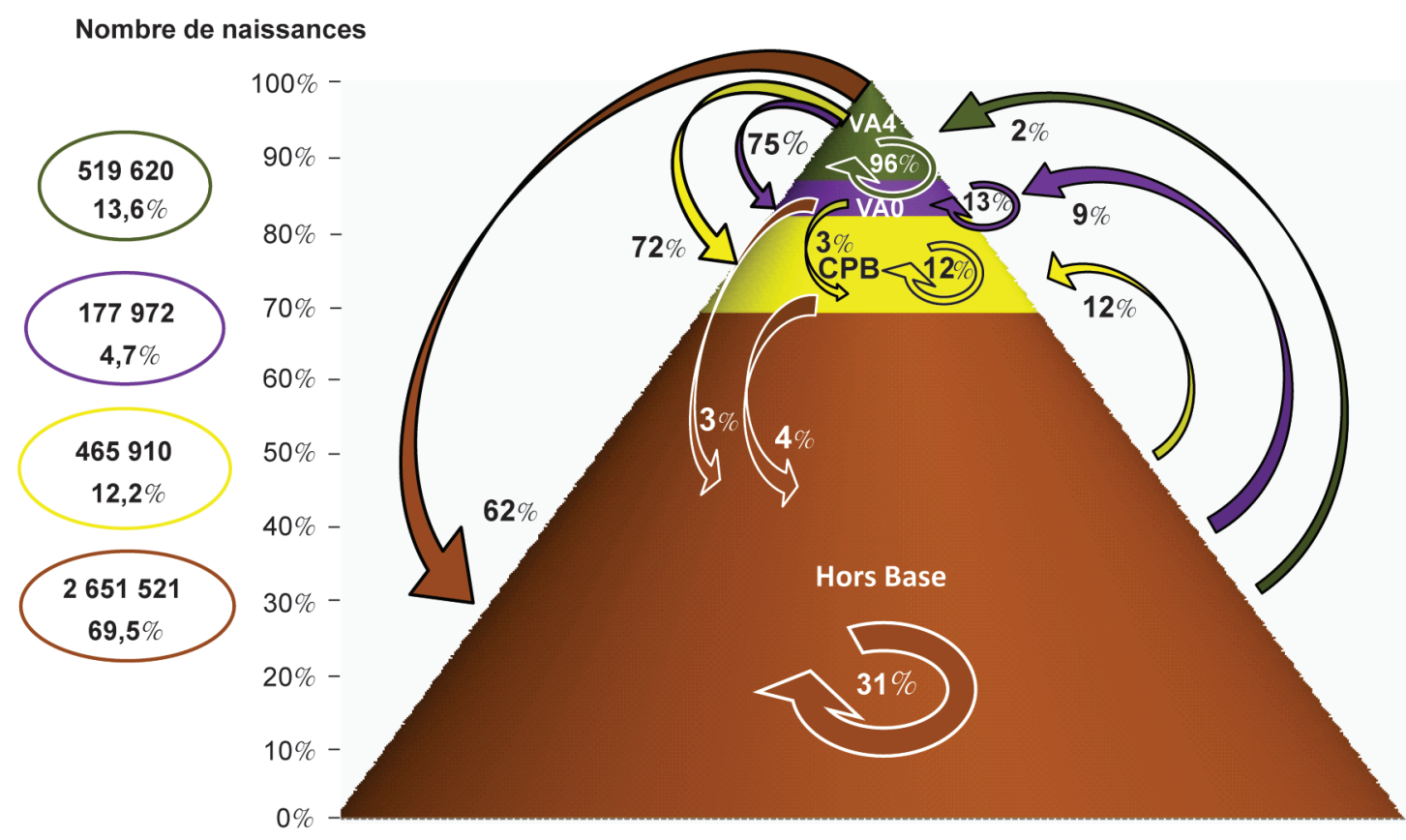

Figure 6. Niveau génétique moyen des pères des veaux nés au cours de la campagne 2015, toutes races confondues.

Index élémentaires exprimés en point (écart-type d'index $=10$ points) (couleur rouge).

Index de synthèse exprimés en point (écart-type d'index $=8$ points) (couleur noire).

Réussite à l'IA : sur insémination animale première en génisse.

Efficacité de carrière : nombre de veaux produits à 6,5 ans.

Caractères bouchers : production de jeunes bovins en ferme.

Le niveau génétique moyen des pères est obtenu en pondérant par le nombre de veaux.

Campagne 2015 : naissances entre le 01/08/14 et le 31/07/15.

Niveau génétique moyen d'ascendance paternelle

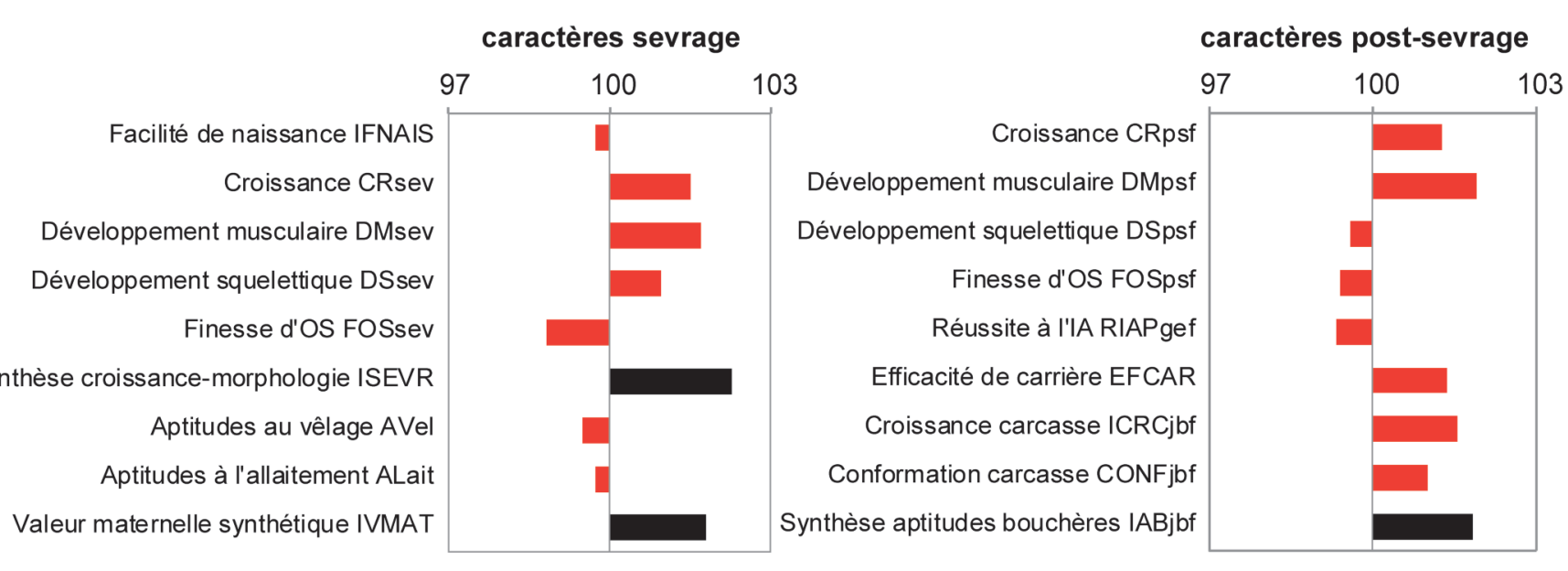

filière en matière de carcasse (maîtrise voire baisse du poids des carcasses) et des orientations raciales choisies par les Organismes de Sélection.

De nouveaux index de synthèse sont ou vont être fournis prochainement, comme par exemple le nouvel index de synthèse de « renouvellement» du troupeau (RENOUV), qui intègre les aptitudes de reproduction («efficacité de carrière », EFCAR), de naissance et de vêlage (IFNAIS et AVel), d'allaitement (ALait), la croissance et la morphologie postsevrage des génisses (CRpsf, DMpsf, DSpsf...).
La dernière évolution majeure en date est la mise en place des évaluations génomiques en 2015. À ce jour, le déploiement de cette méthodologie a été limité aux populations Charolaise, Limousine et Blonde d'Aquitaine et aux caractères pour lesquels les populations de référence disponibles sont de taille suffisante. Les 
index génomiques produits permettent de disposer d'une estimation plus précoce et plus précise de la valeur génétique des animaux comparativement aux index polygéniques, en particulier pour les jeunes animaux. Ce gain de précision, actuellement modeste pour les aptitudes maternelles et les index de croissance et carcasse en jeunes bovins, augmentera au cours du temps, à mesure que les populations de référence s'enrichiront à travers le phénotypage de nouveaux animaux génotypés. L'extension des évaluations génomiques aux autres caractères et populations allaitantes est un objectif. Elle se fera en fonction de l'évolution de la taille des populations de référence disponibles. La mise en place dans les races de plus faibles effectifs reste toutefois un défi compte tenu de la difficulté d'y constituer des populations de référence suffisamment grandes pour calculer des index génomiques précis. Dans ce cas, le génotypage des animaux pour un plus grand nombre de marqueurs génétiques, couplé à l'utilisation de populations de référence élargies en regroupant des animaux de plusieurs races suffisamment proches d'un point de vue génétique pour que les associations entre QTL et marqueurs y soient conservées est une piste à envisager (Hayes et al 2009). Le remplacement de l'actuelle méthode d'évaluation en plusieurs étapes par d'autres méthodes plus optimales est à l'étude. En particulier, la méthode dite « GBLUP en une étape » (ou « singlestep GBLUP », Aguilar et al 2010) semble prometteuse et pourrait permettre de faire bénéficier également les individus non génotypés d'un gain de précision. La prise en compte explicite des mutations causales et QTL détectés dans les programmes de recherche afin d'améliorer la qualité des prédictions est également un objectif.

Une autre perspective liée à l'arrivée de la sélection génomique est l'abandon, comme en bovins laitiers, du testage sur descendance. Cette démarche a déjà été entreprise pour la race Charolaise avec la fin du contrôle individuel et du testage sur descendance en ferme des qualités maternelles fin 2015. Cette épreuve de la descendance en station des qualités maternelles perdure en Limousine et en Blonde d'Aquitaine.

La sélection génomique permet par ailleurs d'envisager le développement de nouvelles évaluations pour des caractères difficiles (ouverture pelvienne) et/ou coûteux à collecter (efficacité alimentaire), et pour lesquels il était donc difficile d'envisager une sélection reposant sur les outils et méthodes classiques. Pour cela, la constitution des populations de référence pourrait passer par la contractualisation de collectes de données spécifiques à travers des réseaux de fermes (ex. ouverture pelvienne) ou le développement de collaboration internationale, à l'image de la collaboration mise en place dans le cadre du programme « global Dry Matter Initiative » (gDMI, de Haas et al 2015).

Enfin, le déploiement du règlement zootechnique européen 2016/1012 du 8 juin 2016 est également à prendre en compte dans les perspectives : ce règlement, en application à partir du $1^{\mathrm{er}}$ novembre 2018, transfère la responsabilité des évaluations génétiques et de la diffusion des résultats de ces évaluations aux Organismes de Sélection raciaux. Ainsi, les évaluations génétiques actuellement confiées à l'INRA par le Ministère chargé de l'Agriculture, pourront être réalisées par chacun des Organismes de Sélection agréés ou déléguées. Il en est de même pour la diffusion des index officiels actuellement définie par France Génétique Élevage et confiée à l'Institut de l'Élevage. De nouveaux opérateurs pourraient ainsi intervenir au niveau des évaluations génétiques officielles en France, mettant ainsi fin au rôle central de l'INRA (évaluations génétiques) et de l'Institut de l'Élevage (diffusion), source importante de mutualisation entre les races.

\section{Conclusion}

La France a su mettre en place au cours des dernières décennies un dispositif génétique collectif performant s'appuyant sur un important contrôle de performances en fermes homogène entre races et sur des stations de contrôles (individuels ou sur descendance) des reproducteurs. $\mathrm{La}$ valorisation de toutes ces informations a été permise par le développement d'évaluations génétiques prenant en compte les spécificités des productions allaitantes (avec notamment les effets maternels sur les performances) et les différentes avancées technologiques (comme l'utilisation de l'information génomique par exemple). L'analyse des résultats sur les caractères au sevrage montre un net progrès génétique sur la croissance et la morphologie des animaux selon les objectifs des races, mais aussi un alourdissement des veaux à la naissance et pour certaines races, une dégradation de la facilité de naissance.

Limitées tout d'abord aux caractères mesurés au sevrage, les évaluations génétiques nationales IBOVAL ont été étendues ces dernières années à de nombreux autres caractères post-sevrage (croissance et morphologie post-sevrage, vie productive, caractères collectés à l'abattage...). Toutes ces nouvelles informations associées aux développements de la sélection génomique permettront dans un avenir proche une mise à jour des objectifs de sélection des bovins allaitants pour prendre en compte les orientations futures de l'élevage et des filières. Le vaste panel d'outils génétiques déjà développés, en cours de développement et à venir restera le socle indispensable à l'adaptation de la filière et de ses éleveurs à leur environnement actuel et futur.

\section{Remerciements}

Nous remercions Gilles Renand et Jean-Luc Brunet pour l'aide fournie lors de la rédaction de cet article. Nous remercions également l'ensemble des collègues ayant par le passé contribué à la mise en place du dispositif français d'amélioration génétique des bovins allaitants, notamment pour les plus récents, Florence Phocas, Marie-Noëlle Fouilloux, Denis Laloë et Gilles Renand. Enfin, nous remercions Normabev pour la fourniture annuelle des données nécessaires aux évaluations sur les aptitudes bouchères. 
Glossaire.

\begin{tabular}{|c|c|}
\hline AF & Note composite des Aptitudes Fonctionnelles \\
\hline CACO & Critère d'Admission au rang des troupeaux COnnectés \\
\hline CD & Coefficient de Détermination \\
\hline $\mathrm{Cl}$ & Station de Contrôle Individuel de taureaux \\
\hline CPB & Certification de la Parenté Bovine, service proposé par les Etablissements d'Elevage \\
\hline DGV & « Direct Genomic Value » : valeur génomique directe \\
\hline DM & Note composite du Développement Musculaire \\
\hline DS & Note composite du Développement Squelettique \\
\hline GEBV & " Genomic Enhanced Breeding Value » : combinaison de la valeur génomique directe et de l'index polygénique \\
\hline IA & Insémination Animale \\
\hline IBOVAL & Indexation BOVine ALlaitante : évaluation génétique des bovins allaitants exploitant les données collectées en ferme \\
\hline IMOCR & Index de synthèse MOrphologie - CRoissance en station \\
\hline IVMAT & Index de Valeur MATernelle synthétique au sevrage \\
\hline MN & Monte Naturelle \\
\hline P120 & Poids à âge type 120 jours \\
\hline $\mathrm{P} 12 \mathrm{M}$ & Poids à âge type 12 mois \\
\hline P18M & Poids à âge type 18 mois \\
\hline P210 & Poids à âge type 210 jours \\
\hline $\mathrm{P} 24 \mathrm{M}$ & Poids à âge type 24 mois \\
\hline PR & Population de Référence \\
\hline psf & Suffixe ajouté aux index des caractères collectés en ferme au niveau du post-sevrage \\
\hline QTL & «Quantitative Trait Loci » : loci de caractères quantitatifs \\
\hline SE & Station d'Evaluation de taureaux \\
\hline SNP & «Single Nucleotide Polymorphism » : le polymorphisme nucléotidique ou polymorphisme d'un seul nucléotide \\
\hline VAO & Service « vaches Allaitantes sans pesée » proposé par les organismes « Bovins Croissance » \\
\hline VA4 & Service "vaches Allaitantes avec pesés » proposé par les organismes « Bovins Croissance » \\
\hline VPS & Service « vaches allaitantes Post-Sevrage » proposé par les organismes « Bovins Croissance » \\
\hline
\end{tabular}

\section{Références}

Aguilar I., Misztal I., Johnson D.L., Legarra A., Tsuruta S., Lawlor T.J., 2010. Hot topic: a unified approach to utilize phenotypic, full pedigree, and genomic information for genetic evaluation of Holstein final score. J. Dairy Sci., 93, 743-752.

Boichard D., Grohs C., Danchin-Burge C., Capitan A., 2016. Les anomalies génétiques: définition, origine, transmission et évolution, mode d'action. In : Anomalies génétiques. Boichard D. (Ed). Dossier, INRA Prod. Anim., 29, 297-306

De Haas Y., Pryce J.E., Calus M.P.L., Wall E., Berry D.P., Løvendahl P., Krattenmacher N., Miglior F., Weigel K., Spurlock D., Macdonald K.A., Hulsegge B., Veerkamp R.F., 2015. Genomic prediction of dry matter intake in dairy cattle from an international data set consisting of research herds in Europe, North America, and Australasia. J. Dairy Sci., 98, 6522-6534.

Ducrocq V., 1992. Les bases de la génétique quantitative. Du modèle génétique au modèle statistique. In : Hors-série, Eléments de génétique quantitative et application aux populations animales. Bibé B., Bonaïti B., Elsen J.M., Guérin G., Mallard J., Minvielle F., De Mondini L., Mulsant P., De Rochambeau H., Farce M.H. (Eds). INRA Prod. Anim., 75-81.

FranceAgriMer, 2016. PCM (Pesée, Classement, Marquage) : guide technique et réglementaire, édition octobre 2016. http://www.franceagrimer.fr/ content/download/48869/469028/file/Guide\% 20PCM\%20VF.pdf

Garrick D.J., Taylor J.F., Fernando R.L., 2009 Deregressing estimated breeding values and weighting information for genomic regression analyses. Genet. Select. Evol., 41, 55.

Grohs C., Duchesne A., Floriot S., Deloche M.C., Boichard D., Ducos A., Danchin-Burge C., 2016. L'Observatoire National des Anomalies Bovines, son action et ses résultats pour une aide efficace à la gestion des anomalies génétiques. In : Anomalies génétiques. Boichard D. (Ed). Dossier, INRA Prod. Anim., 29, 307-318.
Guerrier J., Experton C., Patin S., Phocas F., 2013. Projet OSIRIS : Objectifs de Sélection Innovants en Ruminants et Indices de Synthèse, Renc. Rech. Rum., 20, 142.

Habier D., Fernando R.L., Kizilkay K., Garrick D.J., 2011. Extension of the bayesian alphabet for genomic selection. BMC Bioinformatics, $12,186$.

Hayes B., Bowman P.J., Chamberlain A.C., Verbyla K., Goddard M.E., 2009. Accuracy of genomic breeding values in multi-breed dairy cattle populations. Genet. Select. Evol., 41, 51.

Henderson C.R., 1975. Best linear unbiased estimation and prediction under a selection model. Biometrics, 31, 423-447.

Institut de l'Élevage, 2008. Certification de la parenté des bovins : mode d'emploi de la partie certification du document de notification. http://idele.fr/domaines-techniques/tracabiliteet-certification/tracabilite-des-animaux/publication/idelesolr/recommends/certification-de- 
la-parente-des-bovins-mode-demploi-de-la-partiecertification-du-document-de-no.htm

Institut de l'Élevage, 2013. La Mesure du tour de poitrine : un nouvel outil pour les éleveurs ne pouvant pas peser leurs veaux à la naissance. http://idele.fr/no cache/recherche/publication/i delesolr/recommends/la-mesure-du-tour-depoitrine.htm

Institut de l'Élevage, 2014. Guide pratique du pointage des bovins de race à viande, du sevrage à l'âge adulte. CR n0014201001.

Institut de l'Élevage, 2016. Chiffres-clés Bovins Lait et Viande 2016. http://idele.fr/no cache/ recherche/publication/idelesolr/recommends/ chiffres-cles-bovins-lait-et-viande-2016.html

Institut de l'Élevage, 2017. Indicateurs flux de reproducteurs BV - campagne 2015. Mars 2017. http://idele.fr/domaines-techniques/ameliorerle-troupeau/choix-des-reproducteurs/publication/idelesolr/recommends/indicateurs-fluxde-reproducteurs-bv-campagne-2015-1.html

Institut de l'Élevage et INRA, 2002. Note IBOVAL 28 - mai 2002 - Évolution de la méthode d'appréciation de la connexion entre troupeaux. http://idele.fr/no cache/recherche/publication/ idelesolr/recommends/evolution-de-la-methode -dappreciation-de-la-connexion-entre-troupeaux html

Institut de l'Élevage et INRA, 2005. Note IBOVAL 42 - avril 2005. Améliorations du calcul du CACO en 2005

http://idele.fr/no cache/recherche/publication/i delesolr/recommends/ameliorations-du-calculdu-caco-en-2005.html

Institut de l'Élevage et INRA, 2014. Note IBOVAL 67 - Janvier 2014 - Première publication d'index de morphologie post-sevrage en ferme. http://idele.fr/domaines-techniques/ameliorerle-troupeau/evaluation-genetique/ publication/idelesolr/recommends/premierepublication-dindex-de-morphologie-postsevrage-en-ferme.html.

Meuwissen T.H.E., Hayes B.J., Goddard M.E., 2001. Prediction of total genetic value using genome-wide dense marker maps. Genetics, 157, 1819-1829.

Quaas R.L., Pollack E.J., 1980. Mixed mode methodology for farm and ranch beef cattle testing programs. J. Anim. Sci., 51, 1277-1287.

Tribout T., Gunia M., Saintilan R., Venot E., Fouilloux M.N., Phocas F., 2014. Accuracy of genomic prediction in French Charolais cattle population using high-density chip. In: Proc. $10^{\text {th }}$ WCGALP, Vancouver, Canada.

Tribout T., Barbat M., Saintilan R., Fouilloux M.N., Venot E., Phocas F., 2015. Mise en place d'évaluations génomiques nationales dans les populations bovines allaitantes françaises Charolaise, Limousine et Blonde d'Aquitaine. Renc. Rech. Rum., 22, 103-106.

VanRaden P.M., Van Tassel C.P., Wiggans G.R., Sonstegard T.S., Schnabel R.D., Taylor J.F., Schenkel F.S., 2009. Invited review: Reliability of genomic predictions for North American Holstein bulls. J. Dairy Sci., 92, 16-24.

Venot E., Pabiou T., Hjerpe E., Nilforooshan M.A., Launay A., Wickham B., 2014. Benefits of Interbeef international genetic evaluations for weaning weigth. In: Proc. $10^{\text {th }}$ WCGALP, Vancouver, Canada.

Venot E., Guerrier J., Lajudie P., Dufour V., Leudet O., Boivin X., Sapa J., Phocas F., 2015. Mise en place d'une évaluation nationale du comportement des bovins allaitants à partir de données recueillies en ferme. Renc. Rech. Rum., $22,107-110$

\section{Résumé}

Hérité de la loi sur l'Élevage de 1966, le dispositif génétique français a permis la mise en place d'un vaste recueil de phénotypes en ferme et en station. Toutes ces collectes ont pu être valorisées collectivement au travers de nombreuses évaluations génétiques, et notamment les évaluations nationales sur les données recueillies en ferme appelées «IBOVAL ». Ces évaluations ont évolué tant d'un point de vue méthodologique (évaluations polygéniques et maintenant génomiques) que sur l'éventail des caractères valorisés. La filière de production de viande bovine dispose aujourd'hui d'outils génétiques performants permettant d'évaluer les reproducteurs bovins allaitants, de les sélectionner sur leurs aptitudes bouchères et leur qualités maternelles en ferme et en station (contrôle individuel ou sur descendance). Le panel de caractères traités (naissance, sevrage, post-sevrage, reproduction, aptitudes bouchères) permet d'élaborer des objectifs de sélection adaptés aux orientations raciales, aux contraintes de la filière et de l'élevage. Les programmes de sélection utilisant ces outils génèrent un progrès génétique. Celui-ci est diffusé efficacement, même si la faible pénétration de l'insémination animale reste un facteur limitant. Enfin, l'arrivée de la génomique, les changements organisationnels induits par le nouveau règlement zootechnique européen et le contexte difficile de l'élevage vont entraîner des évolutions au niveau des outils et des objectifs de sélection.

\section{Abstract}

\section{Genetic selection of beef breeds in France: a scheme and a range of innovative tools supporting the beef cattle industry}

The French genetic improvement organisation of beef cattle, the legacy of the French Livestock act of 1966, produces a collection of a wide range of phenotypes obtained on farm and in testing stations. With these, numerous genetic evaluations were developed, in particular evaluations based on data collected on farms called "IBOVAL". The methodology used (with recently the inclusion of genomic information) as well as the number of traits evaluated have evolved with time. Today, adequate genetic tools are available in France to predict genetic values of breeding animals, to select them on their fattening abilities or their maternal abilities. The range of traits (at birth, weaning, post-weaning as well as reproductive and fattening abilities) allows the development of breeding goals adapted to the farmers' needs, the breed objectives and the context of the beef industry. The selection programs using these tools do generate genetic gains. The dissemination of these gains is efficient even though the beef cattle sector has a low rate of insemination. With the introduction of genomics and the organisational changes resulting from the new European regulation as well as the difficult situation of the beef industry, these genetic tools and breeding goals will continue to evolve.

GRIFFON L., BOULESTEIX P., DELPEUCH A., GOVIGNON-GION A., GUERRIER J., LEUDET O., MILLER S., SAINTILAN R., VENOT E., TRIBOUT T., 2017. La sélection génétique des races bovines allaitantes en France : un dispositif et des outils innovants au service des filières viande. In : Élevage bovin allaitant. Agabriel J., Renand G., Baumont R. (Eds). Dossier, INRA Prod. Anim., 30, 107-124. 